\title{
Dynamics and Control of a Planar Multibody Mobile Robot for Confined Environment Inspection
}

\author{
Lounis Douadi \\ School of Electrical Engineering \\ and Computer Science \\ University of Ottawa \\ Email: Idouadi@uottawa.ca
}

\author{
Davide Spinello* \\ Department of Mechanical Engineering \\ University of Ottawa \\ Email: dspinell@uottawa.ca
}

\author{
Wail Gueaieb \\ School of Electrical Engineering \\ and Computer Science \\ University of Ottawa \\ Email:wgueaieb@eecs.uottawa.ca
}

\begin{abstract}
In this paper, we study the dynamics of an articulated planar mobile robot for confined environment exploration. The mobile vehicle is composed of $n$ identical modules hitched together with passive revolute joints. Each module has the structure of a four-bar parallel mechanism on a mobile platform. The dynamic model is derived using Lagrange formulation. Computer simulations illustrate the model by addressing a path following problem inside a pipe. The dynamic model presented in this paper is the basis for the design of motion control algorithms that encode energy optimization and sensor performance maximization.
\end{abstract}

\section{Introduction and Related Literature}

This work is part of a project aimed at the development of a three-dimensional autonomous articulated mobile robot for the exploration and nondestructive testing in confined environments that can be hazardous or not accessible to humans. One application targeted by this system is natural gas pipelines inspection. Articulated mobile robots made their appearance in the framework of service robotics [1]. Snake-like mobile robots are often chosen for the class of applications we are interested in because of their great agility and high redundancy, which enable them to operate in environments that might be too challenging for a conventional wheeled robot. For instance, they are conveniently deployed inside pipelines [2], in narrow spaces, and on the rubble of an earthquake or a major fire, and find applications in fields as diverse as rescue operations, military/defense, and confined environment exploration as, for example, inspection of bridges [3], inspection of natural gas pipelines, industrial pipes and sewer pipelines [4-9], to name a few. Snake-like robots proposed in the literature are typically locomoted either with passive caster wheels supporting their frames [10-12] or with no wheels at all [13-16].

*Address all correspondence to this author. 
For service applications such as inspection and maintenance in confined, not accessible, and eventually hazardous environments it is crucial to equip the robot with decision making and control algorithms that, among other features, maximize the time in which the robot can operate autonomously. This requires the development of dynamic models that account for salient features of the system, and therefore be able to formulate with relatively high accuracy the control efforts involved in typical operating scenarios $[17,18]$. In this way one can optimize the energy consumption and eventually the sensors performance, which is a metric to measure the quality of the inspection and eventual detection of defects and other features. In [19] it is investigated the static and stiffness model of serial-parallel manipulators. In [20] the dynamic model of an articulated vehicle consisting of one car-like tractor and two cart-like semi-trailers is developed using the Newton-Euler equations with the constraint of no slippage. The dynamics of the snake-like robot SES-1 with high speed and high efficiency locomotion is derived in [21]. Transeth et al. [22, 23] presented a three-dimensional wheel-less snake-like robot modeled using nonsmooth dynamics and convex analysis for the incorporation of contact and friction forces. The authors derived a simplified model of planar snake robot locomotion in [24] based on Newton's formulation. The simplification is obtained by considering the body shape changes of the snake robot as linear displacements of the links with respect to each other instead of rotational displacements. A hybrid model of the dynamics of a planar snake robot interacting with obstacles is derived in [25]. The concept of equality of measures is used in [26] to describe the dynamics of a planar snake-like robot while a new dynamic modeling method for snake-like robots using the concept of Nominal Mechanism is introduced in [27]. A recent survey on snake-like robots modeling is given in [28]. The common point between these models is that they deal with the dynamics of multibody systems [29]. Multibody dynamics modeling and simulation has applications in robotics, biomechanics, and virtual reality among others. The major difficulty in modeling multibody systems is the systematic enforcement of internal and external kinematic constraints.

Here, we consider the model of a multibody articulated robot composed of a sequence of modules in plane motion hitched together with passive joints. Each module is a parallel robot on a mobile platform, where a body standing for a payload is supported by two arms with one extremity in contact with the walls of the environment in which the robot operates. The mechanism proposed here is characterized by a higher number of degrees of freedom as compared to other articulated robots, such as the $n$-trailer system, that are often adopted for similar applications. The augmented number of minimal coordinates and increased robot's dexterity and maneuverability inside the pipeline may be crucial in executing some pipe inspection maneuvers. A larger number of available control inputs implies indeed increased versatility in operations that involve for example turning relatively sharp elbows while maintaining the modules centered for the purpose of maximizing sensor performances. We derive the dynamics of the system by considering the Lagrangian formulation, which allows to easily include the internal constraints and it is therefore useful to systematically treat multibody systems. We introduce a non-minimum coordinates set along with a suitable set of constraints stating the couplings among coordinates. The constrained equations of motion form a system of differential algebraic equations. Many methods to solve those equations can be found in the literature [30,31]. We use the method that through differentiation transforms the constraints into second order equations. The shortcoming of this method is that the constraints are enforced at the acceleration level and therefore it is necessary to explicitly check that the corresponding velocity and/or position constraints are satisfied. This motivates works on constraint stabilization [32-34], constraints enforcement and constraint elimination. A survey on special techniques to enforce constraints can be found in [35].

The rest of the paper is organized as follows. In Section 2, we present the structure of the robot. The kinematics is discussed in Section 3, along with singular configurations of the modules. The dynamics and the derivation of the equations of motion of the system are presented in Section 4. The model is illustrated through the simulation of path following control inside a pipe in Section 5. Section 6 is left 
for conclusions.

\section{The Articulated Mobile Robot and The Geometric Description of The Workspace}

In this section, we briefly describe the structural architecture of the robot and the environment in which it operates. As sketched in Figure 1, the vehicle is composed of $n$ identical modules, each forming a closed kinematic chain moving in a connected region $\Omega \in \mathbb{R}^{2}$. The leading and terminal modules $M_{1}$ and $M_{n}$ are called respectively the head and the tail of the robot. The modules are hitched together by $n-1$ passive revolute joints. The name convention for these joints is such that joint $P_{i}$ connects modules $M_{i-1}$ and $M_{i}$ with $i=2 \ldots n$. The vehicle is propelled by $n$ pairs of horizontal active wheels, that are in contact with the walls. In simulation, the contact is enforced by a suitable set of Lagrange multipliers that represent the contact forces between the wheels and the walls. By considering a single module as shown in the schematics of Figure 6, the mechanism can be treated as a closed kinematic chain on a mobile platform. It is similar to a four-bar linkage with the particularity of having mobile bases since the wheels move independently from each other along two curves representing the boundaries of the confined environment. This adds two degrees of freedom as compared to the one-degree-of-freedom four-bar linkage. Hence, a module $M_{i}$, when disconnected, has three degrees of freedom.

A pair of points $P$ and $Q$ in $\Omega$ is mapped into the vector $\mathbf{r}_{P Q}$ by the difference

$$
Q-P=\mathbf{r}_{P Q}
$$

defined as in the usual framework of affine spaces. By referring the space to a global Cartesian reference frame $\left\{O, \mathbf{e}_{x}, \mathbf{e}_{y}\right\}$, with origin $O$ and unit vectors $\mathbf{e}_{x}$ and $\mathbf{e}_{y}$, the difference $P-O$ is the position vector $\mathbf{r}_{O P}$ with representation

$$
\mathbf{r}_{O P}=x_{P} \mathbf{e}_{x}+y_{P} \mathbf{e}_{y}
$$

in the global frame. By referring to Figure $6, h$ and $W$ are respectively the height and the width of the module; $\gamma$ is a nondimensional parameter that represents the fraction of the height of the main body at which the arms are pinned; $L$ is the length of the arms; and $\theta_{i}, \alpha_{i}^{r}$ and $\alpha_{i}^{l}$ are respectively the orientation angle of the module, and the right and left relative joint angles of the right and left shoulder joints. Each pin connecting two modules corresponds to two scalar constraints. For a vehicle with $n$ modules (see Figure 1) the total number of degrees of freedom is $3 n-2(n-1)=n+2$.

In a two-dimensional setting, the right and the left walls of the pipeline are described by the parametric curves $\Pi^{r}$ and $\Pi^{l}$ respectively. Parametric curves evaluated at the arclength $s^{r}$ and $s^{l}$ map to points in the plane, that with a slight abuse of notation can be expressed as position vectors in the global frame $\left\{O, \mathbf{e}_{x}, \mathbf{e}_{y}\right\}$

$$
\begin{aligned}
\Pi^{r}\left(s^{r}\right) & =\Pi_{x}^{r}\left(s^{r}\right) \mathbf{e}_{x}+\Pi_{y}^{r}\left(s^{r}\right) \mathbf{e}_{y} \\
\Pi^{l}\left(s^{l}\right) & =\Pi_{x}^{l}\left(s^{l}\right) \mathbf{e}_{x}+\Pi_{y}^{l}\left(s^{l}\right) \mathbf{e}_{y}
\end{aligned}
$$

with the implicit understanding that position vectors and related Cartesian coordinates $\left\{\Pi_{x}^{l}, \Pi_{y}^{l}\right\}$ and $\left\{\Pi_{x}^{r}, \Pi_{y}^{r}\right\}$ are referred to the origin $O$. The Frenet frames $\left\{\mathbf{T}^{r}, \mathbf{N}^{r}\right\}$ and $\left\{\mathbf{T}^{l}, \mathbf{N}^{l}\right\}$ associated to the parametric curves are defined in terms of the tangent and normal vectors as

$$
\mathbf{T}^{r}=\frac{\mathrm{d} \Pi^{r}}{\mathrm{~d} s^{r}}=\frac{\mathrm{d} \Pi_{x}^{r}}{\mathrm{~d} s^{r}} \mathbf{e}_{x}+\frac{\mathrm{d} \Pi_{y}^{r}}{\mathrm{~d} s^{r}} \mathbf{e}_{y}=: T_{x}^{r} \mathbf{e}_{x}+T_{y}^{r} \mathbf{e}_{y}
$$




$$
\mathbf{N}^{r}=\frac{1}{\kappa^{r}} \frac{\mathrm{d} \mathbf{T}^{r}}{\mathrm{~d} s^{r}}=\frac{1}{\kappa^{r}}\left(\frac{\mathrm{d}^{2} \Pi_{x}^{r}}{\mathrm{~d} s^{r^{2}}} \mathbf{e}_{x}+\frac{\mathrm{d}^{2} \Pi_{y}^{r}}{\mathrm{~d} s^{r^{2}}} \mathbf{e}_{y}\right)=: N_{x}^{r} \mathbf{e}_{x}+N_{y}^{r} \mathbf{e}_{y}
$$

where we have introduced the Cartesian components $\left\{T_{x}^{r}, T_{y}^{r}\right\}$ and $\left\{N_{x}^{r}, N_{y}^{r}\right\}$ of the tangent and of the normal fields to the right wall, and the curvature $\kappa^{r}$. By replacing the superscript $r$ with $l$ the corresponding Frenet frame for the left wall is obtained.

\section{Kinematics}

\subsection{Configuration Kinematics}

Since each module is a closed kinematic chain, the position of the center of mass can be equivalently expressed by the following vector relations, see Figure 6

$$
\begin{aligned}
\mathbf{r}_{O G_{i}} & =\mathbf{r}_{O C_{i}^{r}}+\mathbf{r}_{C_{i}^{r} B_{i}^{r}}+\mathbf{r}_{B_{i}^{r} S_{i}^{r}}+\mathbf{r}_{S_{i}^{r} G_{i}} \\
\mathbf{r}_{O G_{i}} & =\mathbf{r}_{O C_{i}^{l}}+\mathbf{r}_{C_{i}^{l} B_{i}^{l}}+\mathbf{r}_{B_{i}^{l} S_{i}^{l}}+\mathbf{r}_{S_{i}^{l} G_{i}}
\end{aligned}
$$

where $S_{i}^{l}$ and $S_{i}^{r}$ are the points defining the left and right shoulders (arms are attached to the body); $C_{i}^{r}$ and $C_{i}^{l}$ are the points on the right and left wheels that are closest to the related walls; $B_{i}^{r}$ and $B_{i}^{l}$ are the centers of the wheels; and $G_{i}$ is the center of mass of the body of the module $M_{i}$. The position of the center of mass $G_{i}$ is expressed in the global reference frame as

$$
\mathbf{r}_{O G_{i}}=x_{G_{i}} \mathbf{e}_{x}+y_{G_{i}} \mathbf{e}_{y}
$$

For each module, we introduce the following rotated basis, that has the meaning of a body reference frame

$$
\left(\begin{array}{ll}
\mathbf{E}_{i x} & \mathbf{E}_{i y}
\end{array}\right)=\left(\begin{array}{ll}
\mathbf{e}_{x} & \mathbf{e}_{y}
\end{array}\right) \mathbf{R}\left(\theta_{i}\right)
$$

where $\mathbf{R}$ is the rotation matrix

$$
\mathbf{R}\left(\theta_{i}\right)=\left(\begin{array}{cc}
\cos \theta_{i} & -\sin \theta_{i} \\
\sin \theta_{i} & \cos \theta_{i}
\end{array}\right)
$$

In this basis, vectors in (6) are expressed as

$$
\begin{array}{r}
\mathbf{r}_{S_{i}^{r} G_{i}}=-H \mathbf{E}_{i x}+\frac{W}{2} \mathbf{E}_{i y} \\
\mathbf{r}_{B_{i}^{r} S_{i}^{r}}=L R\left(\alpha_{i}^{r}\right) \mathbf{E}_{i x} \\
\mathbf{r}_{S_{i}^{l} G_{i}}=-H \mathbf{E}_{i x}-\frac{W}{2} \mathbf{E}_{i y} \\
\mathbf{r}_{B_{i}^{l} S_{i}^{l}}=-L R\left(\alpha_{i}^{l}\right) \mathbf{E}_{i x}
\end{array}
$$

where $H=h(\gamma-1 / 2)$. By using the orthogonality of $\mathbf{R}$ we express the relative position vectors in the global reference frame as

$$
\mathbf{r}_{S_{i}^{r} G_{i}}=\left(-\frac{W}{2} \sin \theta_{i}-H \cos \theta_{i}\right) \mathbf{e}_{x}
$$




$$
\begin{aligned}
& +\left(\frac{W}{2} \cos \theta_{i}-H \sin \theta_{i}\right) \mathbf{e}_{y} \\
& \mathbf{r}_{B_{i}^{r} S_{i}^{r}}=L\left(\cos \left(\theta_{i}+\alpha_{i}^{r}\right) \mathbf{e}_{x}+\sin \left(\theta_{i}+\alpha_{i}^{r}\right) \mathbf{e}_{y}\right) \\
& \mathbf{r}_{S_{i}^{l} G_{i}}=\left(\frac{W}{2} \sin \theta_{i}-H \cos \theta_{i}\right) \mathbf{e}_{x} \\
& +\left(-\frac{W}{2} \cos \theta_{i}-H \sin \theta_{i}\right) \mathbf{e}_{y} \\
& \mathbf{r}_{B_{i}^{l} S_{i}^{l}}=-L\left(\cos \left(\theta_{i}+\alpha_{i}^{l}\right) \mathbf{e}_{x}+\sin \left(\theta_{i}+\alpha_{i}^{l}\right) \mathbf{e}_{y}\right)
\end{aligned}
$$

Since the wheels are circular, the minimum distance with the walls is achieved at the point $C_{i}^{r}\left(C_{i}^{l}\right)$, such that the tangent to the wheel at the same point is parallel to $\mathbf{T}^{r}\left(s_{i}^{r}\right)\left(\mathbf{T}^{l}\left(s_{i}^{l}\right)\right)$, respectively, where $s_{i}^{r}$ $\left(s_{i}^{l}\right)$ is the corresponding arclength. Therefore the line joining $C_{i}^{r}\left(C_{i}^{l}\right)$ and its projection to the wall is directed as the normal $\mathbf{N}^{r}\left(s_{i}^{r}\right)\left(\mathbf{N}^{l}\left(s_{i}^{l}\right)\right)$, see Figure 3 . The relative position vectors $\mathbf{r}_{C_{i}^{r} B_{i}^{r}}$ and $\mathbf{r}_{C_{i}^{l} B_{i}^{l}}$ in (6) are therefore given by

$$
\begin{array}{r}
\mathbf{r}_{C_{i}^{r} B_{i}^{r}}=\rho \mathbf{N}^{r}\left(s_{i}^{r}\right) \\
=\left(x_{B_{i}^{r}}\left(s_{i}^{r}\right)-x_{C_{i}^{r}}\left(s_{i}^{r}\right)\right) \mathbf{e}_{x}+\left(y_{B_{i}^{r}}\left(s_{i}^{r}\right)-y_{C_{i}^{r}}\left(s_{i}^{r}\right)\right) \mathbf{e}_{y} \\
\mathbf{r}_{C_{i}^{l} B_{i}^{l}}=-\rho \mathbf{N}^{l}\left(s_{i}^{l}\right) \\
=\left(x_{B_{i}^{l}}\left(s_{i}^{l}\right)-x_{C_{i}^{l}}\left(s_{i}^{l}\right)\right) \mathbf{e}_{x}+\left(y_{B_{i}^{l}}\left(s_{i}^{l}\right)-y_{C_{i}^{l}}\left(s_{i}^{l}\right)\right) \mathbf{e}_{y}
\end{array}
$$

where $\rho$ is the radius of the wheels. The characterization of position vectors in (6) is completed by

$$
\begin{gathered}
\mathbf{r}_{O C_{i}^{r}}=\boldsymbol{\Pi}^{r}\left(s_{i}^{r}\right)+\mathbf{r}_{\Pi^{r}\left(s_{i}^{r}\right) C_{i}^{r}} \\
\mathbf{r}_{O C_{i}^{l}}=\boldsymbol{\Pi}^{l}\left(s_{i}^{l}\right)+\mathbf{r}_{\Pi^{l}\left(s_{i}^{l}\right) C_{i}^{l}}
\end{gathered}
$$

where $\mathbf{r}_{\Pi^{r}\left(s_{i}^{r}\right) C_{i}^{r}}$ and $\mathbf{r}_{\Pi^{l}\left(s_{i}^{l}\right) C_{i}^{l}}$ are gap vectors. Here we set the gap to be zero, which corresponds to contact wheels to walls. This condition can be violated by geometric incompatibility between the robot and the walls, as for example in case of sharp turns not compatible with the length of the modules, or when the walls have geometric singularities as corners that cannot be negotiated smoothly. The second case has been studied in [36], and an optimal control on a modified module with extensible arms has been proposed to navigate across the corner while avoiding singularities. In terms of position kinematics, the contact condition implies

$$
\begin{array}{r}
\mathbf{r}_{O B_{i}^{r}}=\mathbf{r}_{O C_{i}^{r}}+\mathbf{r}_{C_{i}^{r} B_{i}^{r}}=\boldsymbol{\Pi}^{r}\left(s_{i}^{r}\right)+\rho \mathbf{N}^{r}\left(s_{i}^{r}\right) \\
=x_{B_{i}^{r}}\left(s_{i}^{r}\right) \mathbf{e}_{x}+y_{B_{i}^{r}}\left(s_{i}^{r}\right) \mathbf{e}_{y} \\
\mathbf{r}_{O B_{i}^{l}}=\mathbf{r}_{O C_{i}^{l}}+\mathbf{r}_{C_{i}^{l} B_{i}^{l}}=\boldsymbol{\Pi}^{l}\left(s_{i}^{l}\right)-\rho \mathbf{N}^{l}\left(s_{i}^{l}\right) \\
=x_{B_{i}^{l}}\left(s_{i}^{l}\right) \mathbf{e}_{x}+y_{B_{i}^{l}}\left(s_{i}^{l}\right) \mathbf{e}_{y}
\end{array}
$$

Substituting from (14) and (11) into (6), and by projecting along $\mathbf{e}_{x}$ and $\mathbf{e}_{y}$ we obtain the geometric 
closure equations of the module's mechanism

$$
\begin{aligned}
& x_{G_{i}}-x_{B_{i}^{r}}\left(s_{i}^{r}\right)+H \cos \theta_{i}+\frac{W}{2} \sin \theta_{i}-L \cos \left(\theta_{i}+\alpha_{i}^{r}\right)=0 \\
& y_{G_{i}}-y_{B_{i}^{r}}\left(s_{i}^{r}\right)+H \sin \theta_{i}-\frac{W}{2} \cos \theta_{i}-L \sin \left(\theta_{i}+\alpha_{i}^{r}\right)=0 \\
& x_{G_{i}}-x_{B_{i}^{l}}\left(s_{i}^{l}\right)+H \cos \theta_{i}-\frac{W}{2} \sin \theta_{i}+L \cos \left(\theta_{i}+\alpha_{i}^{l}\right)=0 \\
& y_{G_{i}}-y_{B_{i}^{l}}\left(s_{i}^{l}\right)+H \sin \theta_{i}+\frac{W}{2} \cos \theta_{i}+L \sin \left(\theta_{i}+\alpha_{i}^{l}\right)=0
\end{aligned}
$$

The geometric relation between the wheels and the arms to which they are attached is expressed by the following relations

$$
\begin{aligned}
\mathbf{r}_{O A_{i}^{r}}+\mathbf{r}_{A_{i}^{r} B_{i}^{r}} & =\mathbf{r}_{O B_{i}^{r}} \\
\mathbf{r}_{O A_{i}^{l}}+\mathbf{r}_{A_{i}^{l} B_{i}^{l}} & =\mathbf{r}_{O B_{i}^{l}}
\end{aligned}
$$

where $A_{i}^{r}$ and $A_{i}^{l}$ are the centers of mass of the right and of the left arms. The relative vectors in the global basis are given by

$$
\begin{array}{r}
\mathbf{r}_{A_{i}^{r} B_{i}^{r}}=-\frac{L}{2} \mathbf{R}\left(\alpha_{i}^{r}\right) \mathbf{E}_{i x} \\
=-\frac{L}{2}\left(\cos \left(\theta_{i}+\alpha_{i}^{r}\right) \mathbf{e}_{x}+\cos \left(\theta_{i}+\alpha_{i}^{r}\right) \mathbf{e}_{y}\right) \\
\mathbf{r}_{A_{i}^{l} B_{i}^{l}}=\frac{L}{2} \mathbf{R}\left(\alpha_{i}^{l}\right) \mathbf{E}_{i x} \\
=\frac{L}{2}\left(\cos \left(\theta_{i}+\alpha_{i}^{l}\right) \mathbf{e}_{x}+\sin \left(\theta_{i}+\alpha_{i}^{l}\right) \mathbf{e}_{y}\right)
\end{array}
$$

By substituting into (16) and by projecting on the global basis the following set of four scalar constraints is obtained

$$
\begin{aligned}
x_{A_{i}^{r}}-x_{B_{i}^{r}}-\frac{L}{2} \cos \left(\theta_{i}+\alpha_{i}^{r}\right) & =0 \\
y_{A_{i}^{r}}-y_{B_{i}^{r}}-\frac{L}{2} \sin \left(\theta_{i}+\alpha_{i}^{r}\right) & =0 \\
x_{A_{i}^{l}}-x_{B_{i}^{l}}+\frac{L}{2} \cos \left(\theta_{i}+\alpha_{i}^{l}\right) & =0 \\
y_{A_{i}^{l}}-y_{B_{i}^{l}}+\frac{L}{2} \sin \left(\theta_{i}+\alpha_{i}^{l}\right) & =0
\end{aligned}
$$

for $i=1, \ldots, n$, where $x_{A_{i}^{r}}, y_{A_{i}^{r}}, x_{A_{i}^{l}}$ and $y_{A_{i}^{l}}$ are the global coordinates of the centers of mass of the right and left arms respectively.

The connection of two modules $M_{i-1}$ and $M_{i}$ by a revolute joint is expressed by constraints formalizing the contact at point $P_{i}$, see Figure 1

$$
\mathbf{r}_{O G_{i-1}}+\mathbf{r}_{G_{i-1} P_{i}}=\mathbf{r}_{O G_{i}}+\mathbf{r}_{G_{i} P_{i}}
$$


In the global reference frame the relative position vectors are given by

$$
\begin{array}{r}
\mathbf{r}_{G_{i-1} P_{i}}=-L_{b} \mathbf{R}\left(\theta_{i-1}\right) \mathbf{E}_{i-1 x} \\
=-L_{b}\left(\cos \theta_{i-1} \mathbf{e}_{x}+\sin \theta_{i-1} \mathbf{e}_{y}\right) \\
\mathbf{r}_{G_{i} P_{i}}=L_{f} \mathbf{R}\left(\theta_{i}\right) \mathbf{E}_{i x} \\
=L_{f}\left(\cos \theta_{i} \mathbf{e}_{x}+\sin \theta_{i} \mathbf{e}_{y}\right)
\end{array}
$$

with $L_{b}=\left\|\mathbf{r}_{G_{i-1} P_{i}}\right\|$ and $L_{f}=\left\|\mathbf{r}_{G_{i} P_{i}}\right\|$ (see Figure 1). Substitution into (19) gives the scalar position constraints

$$
\begin{gathered}
x_{G_{i-1}}-x_{G_{i}}-L_{b} \cos \theta_{i-1}-L_{f} \cos \theta_{i}=0 \\
y_{G_{i-1}}-y_{G_{i}}-L_{b} \sin \theta_{i-1}-L_{f} \sin \theta_{i}=0
\end{gathered}
$$

for $i=1, \ldots, n-1$.

\subsection{Velocity Kinematics}

Time differentiating equations (15) we obtain the tangent closure kinematic equations

$$
\begin{array}{r}
\dot{x}_{G_{i}}+a_{i}^{r} \dot{\theta}_{i}-\dot{x}_{B_{i}^{r}}-c_{i}^{r} \dot{\alpha}_{i}^{r}=0 \\
\dot{y}_{G_{i}}+b_{i}^{r} \dot{\theta}_{i}-\dot{y}_{B_{i}^{r}}-d_{i}^{r} \dot{\alpha}_{i}^{r}=0 \\
\dot{x}_{G_{i}}+a_{i}^{l} \dot{\theta}_{i}-\dot{x}_{B_{i}^{l}}-c_{i}^{l} \dot{\alpha}_{i}^{l}=0 \\
\dot{y}_{G_{i}}+b_{i}^{l} \dot{\theta}_{i}-\dot{y}_{B_{i}^{l}}-d_{i}^{l} \dot{\alpha}_{i}^{l}=0
\end{array}
$$

for $i=1, \ldots, n$, where $a_{i}^{r}, b_{i}^{r}, c_{i}^{r}, d_{i}^{r}$ and the analogous for the left arm are configuration-dependent parameters listed in the Appendix.

In the velocity space, the permanent contact between the wheels and the walls is formalized by rolling without slipping constraints, which state that the relative velocity between the walls and the contact point is zero:

$$
\begin{aligned}
\dot{\mathbf{r}}_{\boldsymbol{\Pi}^{r}\left(s_{i}^{r}\right) C_{i}^{r}} & =\mathbf{0} \\
\dot{\mathbf{r}}_{\boldsymbol{\Pi}^{l}\left(s_{i}^{l}\right) C_{i}^{l}} & =\mathbf{0}
\end{aligned}
$$

We assume that the walls are at rest, which implies that the absolute velocities $\dot{\mathbf{r}}_{O C_{i}^{r}}$ and $\dot{\mathbf{r}}_{O C_{i}^{l}}$ of the points in contact with the walls are zero. By using the rigid body velocity transformation we obtain

$$
\begin{array}{r}
\dot{\mathbf{r}}_{O C_{i}^{r}}=\dot{\mathbf{r}}_{O B_{i}^{r}}+\boldsymbol{\omega}_{i}^{r w} \wedge \mathbf{r}_{B_{i}^{r} C_{i}^{r}}=\mathbf{0} \\
\dot{\mathbf{r}}_{O C_{i}^{l}}=\dot{\mathbf{r}}_{O B_{i}^{l}}+\boldsymbol{\omega}_{i}^{l w} \wedge \mathbf{r}_{B_{i}^{l} C_{i}^{l}}=\mathbf{0}
\end{array}
$$

where $\dot{\mathbf{r}}_{O B_{i}^{r}}$ and $\dot{\mathbf{r}}_{O B_{i}^{l}}$ are the absolute velocities of the centers of mass of the wheels, $\boldsymbol{\omega}_{i}^{r w}$ and $\boldsymbol{\omega}_{i}^{l w}$ are the angular velocities, and " $\wedge$ " is the exterior (cross) product. By using the representations in (12), the 
projection of (24) on the global basis gives the scalar rolling without slipping relations

$$
\begin{array}{r}
\dot{x}_{B_{i}^{r}}+\rho \omega_{i}^{r w} N_{y}^{r}\left(s_{i}^{r}\right)=0 \\
\dot{y}_{B_{i}^{r}}-\rho \omega_{i}^{r w} N_{x}^{r}\left(s_{i}^{r}\right)=0 \\
\dot{x}_{B_{i}^{l}}-\rho \omega_{i}^{l w} N_{y}^{l}\left(s_{i}^{l}\right)=0 \\
\dot{y}_{B_{i}^{l}}+\rho \omega_{i}^{l w} N_{x}^{l}\left(s_{i}^{l}\right)=0
\end{array}
$$

for $i=1, \ldots, n$. We note that the rolling without slipping constraint implies that the two components of the absolute velocity of the contact points are zero (since the walls are at rest). Therefore, by choosing an initial configuration in which the wheels are in contact with the walls, this constraint is sufficient to ensure that they remain in contact as the system evolves. Since the angular velocity vectors are normal to the plane of the position vectors, the cross products in (24) can be written in terms of the Frenet frames as

$$
\begin{gathered}
\boldsymbol{\omega}_{i}^{r w} \wedge \mathbf{r}_{B_{i}^{r} C_{i}^{r}}=\boldsymbol{\omega}_{i}^{r w} \wedge\left(-\rho \mathbf{N}^{r}\right)=\rho \omega_{i}^{r w} \mathbf{T}^{r} \\
\boldsymbol{\omega}_{i}^{l w} \wedge \mathbf{r}_{B_{i}^{l} C_{i}^{l}}=\boldsymbol{\omega}_{i}^{l w} \wedge \rho \mathbf{N}^{l}=-\rho \omega_{i}^{l w} \mathbf{T}^{l}
\end{gathered}
$$

Moreover, time differentiating expressions in (14), and by using the second Frenet-Serret formula we obtain

$$
\begin{gathered}
\dot{\mathbf{r}}_{O B_{i}^{r}}=\dot{\mathbf{\Pi}}^{r}\left(s_{i}^{r}\right)+\rho \dot{\mathbf{N}}^{r}\left(s_{i}^{r}\right)=\dot{s}_{i}^{r} \mathbf{T}^{r}\left(s_{i}^{r}\right)-\rho \dot{s}_{i}^{r} \kappa^{r} \mathbf{T}^{r}\left(s_{i}^{r}\right) \\
\dot{\mathbf{r}}_{O B_{i}^{l}}=\dot{\boldsymbol{\Pi}}^{l}\left(s_{i}^{l}\right)-\rho \dot{\mathbf{N}}^{l}\left(s_{i}^{l}\right)=\dot{s}_{i}^{l} \mathbf{T}^{l}\left(s_{i}^{l}\right)+\rho \dot{s}_{i}^{l} \kappa^{l} \mathbf{T}^{l}\left(s_{i}^{l}\right)
\end{gathered}
$$

which shows that rolling without slipping constraints imply that velocities of the center of mass of the wheels are parallel to the tangent to the wall at the contact points. By combining (27), (26), and (24) we obtain the following relations between the wheels angular velocities and the rate of change of the arclength parameters

$$
\begin{gathered}
\dot{s}_{i}^{r}=-\frac{\rho}{1-\rho \kappa^{r}} \omega_{i}^{r w} \\
\dot{s}_{i}^{l}=\frac{\rho}{1+\rho \kappa^{l}} \omega_{i}^{l w}
\end{gathered}
$$

Therefore the change of arclength and the angular velocity can be considered, from a kinematic perspective, as driving parameters of the system. The rate of change of the arclength parameters can be regarded as the speed of the wheel's center of mass, that by virtue of the rolling without slipping constraints is related to the angular velocity. When the curvature of the wall is zero the expressions in (28) give the expected relations between angular velocity, forward speed of the center of mass, and radius of the wheel. If the radius of curvature of the wall, $1 / \kappa$, is larger than the radius of curvature of the wheel, $\rho$, then $\dot{s}$ and $\omega$ are consistent with respect to the forward motion of the mechanism; if the radius of curvature of the wall is smaller than the radius of curvature of the wheel then $\dot{s}$ and $\omega$ are not consistent with respect to the forward motion, which means that a forward angular velocity (with respect to the motion) of the wheel corresponds to backward linear velocity (with respect to the motion) of its center 
of mass. Therefore scenarios that involve walls with large curvature require special attention. Note that the signs in (28) account for the fact that, with respect to forward motion, the inner part of the pipeline is to the right of $\Pi^{l}$ and to the left of $\Pi^{r}$.

The set of velocity constraints associated to each module is completed by differentiating (18) in time, to obtain the wheel to arm pinning condition in the velocity space:

$$
\begin{aligned}
\dot{x}_{B_{i}^{r}}-\dot{x}_{A_{i}^{r}}-\frac{L}{2}\left(\dot{\theta}_{i}+\dot{\alpha}_{i}^{r}\right) \sin \left(\theta_{i}+\alpha_{i}^{r}\right) & =0 \\
\dot{y}_{B_{i}^{r}}-\dot{y}_{A_{i}^{r}}+\frac{L}{2}\left(\dot{\theta}_{i}+\dot{\alpha}_{i}^{r}\right) \cos \left(\theta_{i}+\alpha_{i}^{r}\right) & =0 \\
\dot{x}_{B_{i}^{l}}-\dot{x}_{A_{i}^{l}}+\frac{L}{2}\left(\dot{\theta}_{i}+\dot{\alpha}_{i}^{l}\right) \sin \left(\theta_{i}+\alpha_{i}^{l}\right) & =0 \\
\dot{y}_{B_{i}^{l}}-\dot{y}_{A_{i}^{l}}-\frac{L}{2}\left(\dot{\theta}_{i}+\dot{\alpha}_{i}^{l}\right) \cos \left(\theta_{i}+\alpha_{i}^{l}\right) & =0
\end{aligned}
$$

The velocity constraints that express the connection between two modules are obtained by differentiating (21) in time

$$
\begin{array}{r}
\dot{x}_{G_{i-1}}-\dot{x}_{G_{i}}+L_{b} \dot{\theta}_{i-1} \sin \theta_{i-1}+L_{f} \dot{\theta}_{i} \sin \theta_{i}=0 \\
\dot{y}_{G_{i-1}}-\dot{y}_{G_{i}}-L_{b} \dot{\theta}_{i-1} \cos \theta_{i-1}-L_{f} \dot{\theta}_{i} \cos \theta_{i}=0
\end{array}
$$

for $i=1, \ldots, n-1$.

We adopt the following nonminimal set of joint coordinates $q_{i}$ and set of end effector coordinates $p_{i}$ (here we use the term end effector to refer to the platform of the mechanism)

$$
\begin{array}{r}
q_{i}=\left[x_{B_{i}^{r}}, y_{B_{i}^{r}}, x_{B_{i}^{l}}, y_{B_{i}^{l}}, \alpha_{i}^{r}, \alpha_{i}^{l}, \theta_{i}^{r}, \theta_{i}^{l}, x_{A_{i}^{r}}, y_{A_{i}^{r}}, x_{A_{i}^{l}}, y_{A_{i}^{l}}\right] \\
p_{i}=\left[x_{G_{i}}, y_{G_{i}}, \theta_{i}\right]
\end{array}
$$

where $\theta_{i}^{r}$ and $\theta_{i}^{l}$ are the angular coordinates of the wheels. By collectively expressing equations (22), (25), and (29) we rewrite the systems in matrix form as

$$
\mathbf{J}_{p_{i}} \dot{p}_{i}=\mathbf{J}_{q_{i}} \dot{q}_{i}
$$

where $\mathbf{J}_{p_{i}} \in \mathbb{R}^{12 \times 3}$ and $\mathbf{J}_{q_{i}} \in \mathbb{R}^{12 \times 12}$ are, respectively, the parallel and the serial Jacobians of the mechanism and are given in the Appendix.

For a vehicle with $n$ modules, let $p=\left[p_{1} \ldots p_{n}\right]^{\top} \in \mathbb{R}^{3 n}$ be the collection of operational coordinates and $q=\left[q_{1} \ldots q_{n}\right]^{\top} \in \mathbb{R}^{12 n}$ be the collection of joint coordinates. The collective velocity kinematics of the multibody mechanism that include the hitching constraints (30) is therefore written as

$$
\mathbf{J}_{p} \dot{p}=\mathbf{J}_{q} \dot{q}
$$

with Jacobians $\mathbf{J}_{p} \in \mathbb{R}^{(14 n-2) \times 3 n}$ and $\mathbf{J}_{q} \in \mathbb{R}^{(14 n-2) \times 12 n}$ given in the Appendix. Note that the coupling between modules is included in $\mathbf{J}_{p}$.

For the system of $n$ modules with $n-1$ joints we have therefore $12 n+2(n-1)$ constraints among which $12 n$ describe the inner connections among parts of the individual modules (equations (22) and 
(29)) and the rolling without slipping constraint (equations (25)), while the remaining $2 n-2$ describe hitching constraints (equations (30)). The total number of kinematic constraints is therefore $14 n-2$ for $15 n$ unknowns ( $3 n$ Cartesian velocities and $12 n$ joint velocities). This leaves $n+2$ degrees of freedom, which is the dimension of a minimal set of coordinates for the articulated vehicle. For instance, one can choose position and orientation of the head and the $n-1$ orientations, one for each remaining modules.

\subsection{Singularities}

Singularities occur when one or both Jacobians, $\mathbf{J}_{p}$ and $\mathbf{J}_{q}$, become singular [37]. A parallel singularity occurs when the parallel Jacobian $\mathbf{J}_{p}$ takes a singular value, and a serial singularity occurs when the serial Jacobian $\mathbf{J}_{q}$ is singular, corresponding to configurations where the robot loses one or more degrees of freedom [38]. For a single module $M_{i}$, a serial singularity occurs when $\operatorname{det} \mathbf{J}_{q_{i}}=0$. This implies

$$
\begin{array}{r}
N_{x}^{l}\left(s_{i}^{l}\right) \sin \left(\theta_{i}+\alpha_{i}^{l}\right)=N_{y}^{l}\left(s_{i}^{l}\right) \cos \left(\theta_{i}+\alpha_{i}^{l}\right) \\
\text { or } \\
N_{x}^{r}\left(s_{i}^{r}\right) \sin \left(\theta_{i}+\alpha_{i}^{r}\right)=N_{y}^{r}\left(s_{i}^{r}\right) \cos \left(\theta_{i}+\alpha_{i}^{r}\right) .
\end{array}
$$

These conditions correspond to configurations where one or both arms of the module are normal to the corresponding pipe wall. Figure 4 illustrates two examples of such cases. The singular position depicted in figure 4(a) occurs when each arm is perpendicular to the surface it is in contact with. It can be easily avoided by having the mechanism's span to be larger than the pipe diameter. Nevertheless, serial singular configurations as in figure 4(b) are still physically possible. Avoiding them can be encoded as part of the controller's objectives [36].

Parallel singularities occur when the parallel Jacobian $\mathbf{J}_{p}$ is singular, or if it is not of full rank for a non-square Jacobian. For a single module $M_{i}$, we study the rank of $\mathbf{J}_{p i}$ through the eigenvalues of matrix $\mathbf{J}_{p i}^{\top} \mathbf{J}_{p i}$ based on the fact that the singular values of $\mathbf{J}_{p i}$ are the square roots of the eigenvalues of $\mathbf{J}_{p i}^{\top} \mathbf{J}_{p i}$, and by using the fact that the rank of $\mathbf{J}_{p i}$ equals the number of its nonzero singular values [39]. If $\operatorname{det}\left(\mathbf{J}_{p i}^{\top} \mathbf{J}_{p i}\right) \neq 0$, then all its eigenvalues are non-zero, and therefore all the singular values of $\mathbf{J}_{p i}$ are non-zero, which implies that $\mathbf{J}_{p i}$ is full rank. The determinant of $\mathbf{J}_{p i}^{\top} \mathbf{J}_{p i}$ may be expressed as

$$
\begin{array}{r}
\operatorname{det}\left(\mathbf{J}_{p_{i}}^{\top} \mathbf{J}_{p_{i}}\right)= \\
W^{2}+2 L^{2}\left(1+\cos \left(\alpha_{i}^{r}-\alpha_{i}^{l}\right)\right)+2 W L\left(\sin \alpha_{i}^{r}+\sin \alpha_{i}^{l}\right) .
\end{array}
$$

This determinant vanishes when $\alpha_{i}^{r}-\alpha_{i}^{l}=\pi$ and $W=0$. Figure 5 depicts a configuration corresponding to those conditions. We assume that the module width $W$ is nonzero. A way to avoid parallel singularities is to set the constraints $0<\alpha_{i}^{r}<\frac{\pi}{2}$ and $\frac{\pi}{2}<\alpha_{i}^{l}<\pi$ on the shoulder joints, so that the determinant never vanishes.

\section{Dynamics}

\subsection{Equations of Motion}

In this section, we present the dynamics and derive the equations of motion of the system. As discussed in previous sections, each module is a multibody system comprising the main body standing for the payload, two arms and two wheels. The equations of motion of the system are derived based 
on Lagrange's formulation. For each module, we use the non-minimal set of coordinates $\xi_{i}^{\top}=\left[p_{i}^{\top}, q_{i}^{\top}\right]$, with $\xi_{i} \in \mathbb{R}^{15}$ and $p_{i}$ and $q_{i}$ defined in (31). Constraints (22), (25) and (29) are accounted for through Lagrange multipliers. The Lagrangian function $\mathcal{L}_{i}\left(\xi_{i}\right)$ of module $M_{i}$ reduces to its kinetic energy $\mathcal{T}_{i}\left(\xi_{i}\right)$ since we consider a planar system with no potential fields. Contributions to the Lagrangian can be explicitly identified as the individual contributions of the kinetic energies of the parts composing a module

$$
\mathcal{L}_{i}=\mathcal{T}_{i}^{b}+\mathcal{T}_{i}^{r a}+\mathcal{T}_{i}^{l a}+\mathcal{T}_{i}^{r w}+\mathcal{T}_{i}^{l w}
$$

where $\mathcal{T}_{i}^{b}, \mathcal{T}_{i}^{r a}, \mathcal{T}_{i}^{l a}, \mathcal{T}_{i}^{r w}$, and $\mathcal{T}_{i}^{l w}$ are respectively the kinetic energy of the main body (payload or end effector), right arm, left arm, right wheel and left wheel. As illustrated in Figure 6, we consider the main body to be rectangular with mass $m$, the arms as rods of identical mass $m_{a}$, and the wheels as discs with identical mass $m_{w}$. Therefore the kinetic energies written in terms of translational and rotational contributions are given by

$$
\begin{array}{r}
\mathcal{T}_{i}^{b}=\frac{1}{2} \dot{\mathbf{r}}_{G_{i}} \cdot m \dot{\mathbf{r}}_{G_{i}}+\frac{1}{2} I_{b} \dot{\theta}_{i}^{2} \\
\mathcal{T}_{i}^{r a}=\frac{1}{2} \dot{\mathbf{r}}_{A_{i}^{r}} \cdot m_{a} \dot{\mathbf{r}}_{A_{i}^{r}}+\frac{1}{2} \boldsymbol{\omega}_{i}^{r a} \cdot I_{a} \boldsymbol{\omega}_{i}^{r a} \\
\mathcal{T}_{i}^{r w}=\frac{1}{2} \dot{\mathbf{r}}_{B_{i}^{r}} \cdot m_{w} \dot{\mathbf{r}}_{B_{i}^{r}}+\frac{1}{2} \boldsymbol{\omega}_{i}^{r w} \cdot I_{w} \boldsymbol{\omega}_{i}^{r w}
\end{array}
$$

where "." is the inner product; $\dot{\mathbf{r}}_{G_{i}}, \dot{\mathbf{r}}_{A_{i}^{r}}$, and $\dot{\mathbf{r}}_{B_{i}^{r}}$ are the velocities of the centers of mass of the body, right arm, and right wheel (analogous expressions can be obtained for the left arm and wheel by renaming the superscript from $r$ to $l$ ); $\boldsymbol{\omega}_{i}^{r a}$ is the angular velocity of the right arm (normal to the plane defined by the linear velocities); and $I_{b}, I_{a}$, and $I_{w}$ are respectively polar moments of inertia of the main body, right arm, and right wheel about their own centers of mass. Kinetic energy expressions for the left arm and left wheel are obtained by changing the superscript $r$ to $l$ in (38). By using the law of composition of angular velocities (see for example [40, Chapter 4]) and by recalling that $\alpha_{i}^{r}$ is the relative angular position of the arm with respect to the body, the angular velocity of the arm can be written as

$$
\boldsymbol{\omega}_{i}^{r a}=\dot{\theta}_{i} \mathbf{e}_{z}+\dot{\alpha}_{i}^{r} \mathbf{e}_{z}
$$

where $\mathbf{e}_{z}$ is the unit normal to the plane of the motion. By projecting vectors in (38) in the orthonormal basis $\left\{\mathbf{e}_{x}, \mathbf{e}_{y}, \mathbf{e}_{z}\right\}$ we obtain the expressions for the kinetic energies in terms of the adopted set of nonminimal coordinates

$$
\begin{array}{r}
\mathcal{T}_{i}^{b}=\frac{1}{2} m\left(\dot{x}_{G_{i}}^{2}+\dot{y}_{G_{i}}^{2}\right)+\frac{1}{2} I_{b} \dot{\theta}_{i}^{2} \\
\mathcal{T}_{i}^{r a}=\frac{1}{2} m_{a}\left(\dot{x}_{A_{i}^{r}}^{2}+\dot{y}_{A_{i}^{r}}^{2}\right)+\frac{1}{2} I_{a}\left(\dot{\alpha}_{i}^{r}+\dot{\theta}_{i}\right)^{2} \\
\mathcal{T}_{i}^{r w}=\frac{1}{2} m_{w}\left(\dot{x}_{B_{i}^{r}}^{2}+\dot{y}_{B_{i}^{r}}^{2}\right)+\frac{1}{2} I_{w} \omega_{i}^{r w 2}
\end{array}
$$

Given the dynamics of one module, we can derive the dynamics of the articulated mobile robot by incorporating the constraints relating adjacent modules. Hitching $n$ modules together results in a 
more complex constrained multibody system. We define the state vector $\xi=\left[\xi_{1}, \ldots, \xi_{n}\right] \in \mathbb{R}^{15 n}$, that is non-minimal as the number of degrees of freedom of the mechanism is $n+2$.

In order to derive the equations of motion for the multibody system composed of $n$ modules, we consider the Lagrangian of the articulated vehicle that is the sum of Lagrangians of the $n$ modules

$$
\mathcal{L}(\xi)=\sum_{i=1}^{n} \mathcal{L}_{i}\left(\xi_{i}\right)=\sum_{i=1}^{n} \mathcal{T}_{i}\left(\xi_{i}\right)
$$

Since we have chosen a set of nonminimal coordinates that allows us to easily write the kinetic energies, the $12 n$ constraints (12 for each module) (22), (25), and (29) and the $2(n-1)$ hitching constraints (30) must be included in the Lagrangian formulation, for the total number of velocity constraints given by $12 n+2(n-1)=14 n-2$. We collectively express them in matrix form by

$$
\mathbf{C} \dot{\xi}=0
$$

where $\mathbf{C} \in \mathbb{R}^{(14 n-2) \times 15 n}$ is the velocity constraint Jacobian of the multibody system, that is given by

$$
\mathbf{C}=\left[\begin{array}{ccccc}
\mathbf{J}_{1} & & & & \\
\overline{\mathbf{Q}}_{1} & \overline{\mathbf{S}}_{2} & & & \\
& \mathbf{J}_{2} & & 0 & \\
& \overline{\mathbf{Q}}_{2} & \overline{\mathbf{S}}_{3} & & \\
& & \ddots & & \\
& & & \mathbf{J}_{n-1} & \\
& & & \overline{\mathbf{Q}}_{n-1} & \overline{\mathbf{S}}_{n} \\
& & & & \mathbf{J}_{n}
\end{array}\right]
$$

where $\mathbf{J}_{i} \in \mathbb{R}^{12 \times 15}$ is the velocity constraint Jacobian for one module, which is the block matrix given by

$$
\mathbf{J}_{i}=\left[\mathbf{J}_{p_{i}}-\mathbf{J}_{q_{i}}\right]
$$

The serial and parallel Jacobians $\mathbf{J}_{p_{i}}$ and $\mathbf{J}_{q_{i}}$ are defined in (79). Moreover, $\overline{\mathbf{Q}}_{i}$ and $\overline{\mathbf{S}}_{i}$ both in $\mathbb{R}^{2 \times 15}$ are defined based on $\mathbf{Q}_{i}$ and $\mathbf{S}_{i}$ in (80) to account for the pins that connect pairs of modules

$$
\begin{array}{r}
\overline{\mathbf{Q}}_{i}=\left[\mathbf{Q}_{i} 0_{2 \times 12}\right] \\
\overline{\mathbf{S}}_{i}=\left[\mathbf{S}_{i} 0_{2 \times 12}\right]
\end{array}
$$

We denote the vector that collects all multipliers with $\lambda=\left(\lambda_{1}, \bar{\lambda}_{1}, \ldots, \lambda_{n-1}, \bar{\lambda}_{n-1}, \lambda_{n}\right) \in \mathbb{R}^{14 n-2}$, with $\lambda_{i} \in \mathbb{R}^{12}$ and $\bar{\lambda}_{i} \in \mathbb{R}^{2}$ respectively being associated to the modules' constraints (22), (25), and (29), and to the hitching constraints (30). Since the velocity constraints are affine in the velocity we include them in the equations of motion as follows [41]:

$$
\frac{\mathrm{d}}{\mathrm{d} t}\left(\frac{\partial \mathcal{L}}{\partial \dot{\xi}}\right)-\frac{\partial \mathcal{L}}{\partial \xi}+\mathbf{C}^{\top} \boldsymbol{\lambda}=\boldsymbol{\tau}
$$


where $\boldsymbol{\tau}=\left[\boldsymbol{\tau}_{1}, \ldots, \boldsymbol{\tau}_{n}\right] \in \mathbb{R}^{15 n}$ is the vector of torques of the articulated vehicle, with components $\boldsymbol{\tau}_{i}$ representing actuation actions for the $i$-th module

$$
\boldsymbol{\tau}_{i}=\left[0_{1 \times 7}, \tau_{i}^{r s}, \tau_{i}^{l s}, \tau_{i}^{r w}, \tau_{i}^{l w}, 0_{1 \times 4}\right]
$$

Scalars $\tau_{i}^{r s}, \tau_{i}^{l s}, \tau_{i}^{r w}$, and $\tau_{i}^{l w}$ are the torques to the right and left joints of the shoulders and to the right and left wheel shafts respectively. In our case $\frac{\partial \mathcal{L}}{\partial \xi}=0$. The matrix form of the constrained equations of motion gives therefore the following system of differential algebraic equations

$$
\begin{aligned}
\mathbf{M} \ddot{\xi}+\mathbf{C}^{\top} \boldsymbol{\lambda} & =\tau \\
\mathbf{C} \dot{\xi} & =0
\end{aligned}
$$

The linear operator $\mathbf{M} \in \mathbb{R}^{15 n \times 15 n}$ is the block diagonal mass matrix $\mathbf{M}=\operatorname{diag}\left[\mathbf{M}_{1}, \ldots, \mathbf{M}_{n}\right]$ resulting from the additive structure of the Lagrangian function, with contributions $\mathbf{M}_{i} \in \mathbb{R}^{15 \times 15}$ for individual modules given by

$$
\mathbf{M}_{i}=\left[\begin{array}{ccccccc}
m \mathbf{I}_{2} & 0 & 0 & 0 & 0 & 0 & 0 \\
0 & I_{b}+2 I_{a} & 0 & I_{a} & I_{a} & 0 & 0 \\
0 & 0 & m_{w} \mathbf{I}_{4} & 0 & 0 & 0 & 0 \\
0 & I_{a} & 0 & I_{a} & 0 & 0 & 0 \\
0 & I_{a} & 0 & 0 & I_{a} & 0 & 0 \\
0 & 0 & 0 & 0 & 0 & I_{w} \mathbf{I}_{2} & 0 \\
0 & 0 & 0 & 0 & 0 & 0 & m_{a} \mathbf{I}_{4}
\end{array}\right]
$$

where $\mathbf{I}_{2}$ and $\mathbf{I}_{4}$ are identity matrices of the dimension indicated by the subscript, and zeros represent zero matrices of appropriate dimensions, dictated by the consistency condition with the dimensions of the diagonal block.

\subsection{Numerical Integration}

We solve the system (51) by direct elimination of the Lagrange multipliers $[35,42,43]$ which as a consequence reduces the index of the differential algebraic equation from 3 to 1, where the index of a differential algebraic equation may be defined roughly as the number of times the constraints (in our case the geometric constraints) have to be differentiated in order to transform the system of differential algebraic equations into a system of differential equations. By time differentiating the second equation in (51) and by solving the system for $\ddot{\xi}$ and $\lambda$, we find the following explicit expressions for the acceleration and the Lagrange multipliers vectors

$$
\begin{array}{r}
\ddot{\xi}=\mathbf{M}^{-1}\left(\left(\mathbf{I}_{15 n}-\mathbf{C}^{\top} \mathbf{D}^{-1} C \mathbf{M}^{-1}\right) \boldsymbol{\tau}-\mathbf{C}^{\top} \mathbf{D}^{-1} \dot{\mathbf{C}} \dot{\xi}\right) \\
\lambda=\mathbf{D}^{-1}\left(\mathbf{C} \mathbf{M}^{-1} \boldsymbol{\tau}+\dot{\mathbf{C}} \dot{\xi}\right)
\end{array}
$$

with $\mathbf{D} \in \mathbb{R}^{14 n-2 \times 14 n-2}$ given by $\mathbf{D}=\mathbf{C M}^{-1} \mathbf{C}^{\top}$. Equation (53a) is transformed into state-space form by the change of variable $z=\dot{\xi}$, and then integrated numerically using for example the Range-Kutta algorithm. For complex systems, for example involving a large number of degrees of freedom, it is 
appropriate to use advanced $O(N)$ or $O(\log N)$ algorithms [44-46]. This method has the drawback to be potentially unstable if the numerical integration is extended over a relatively long time interval, since the constraints are enforced at the acceleration level and therefore errors can accumulate for the velocity and position. Therefore, the constraints on position and velocity must be enforced explicitly in order to avoid the drift phenomena associated to the propagation of truncation and round-off errors. For a similar approach the reader can refer to $[43,47]$.

\section{Motion Control Inside a Pipe: a Show Case}

Here we present an application of the model presented in previous sections. The objective of this study is to illustrate it through simulations that involve some peculiarity of the scenarios for which our system will be designed and implemented. The environment is a planar strip that models the longitudinal section of a pipeline in which the robot is deployed. We consider a path following kinematic control to track the axis of the pipeline to reproduce the optimal operational condition of several sensors mounted on inspection mobile robots. The path planner is coupled with a PD torque control based on the dynamic model, that estimates the torques (actuators efforts) necessary to track the desired trajectory. The control scheme is sketched in Fig. 6.

\subsection{Path Following using Frenet Frames}

As stated in the Introduction, the main targeted application of the system is pipeline maintenance and inspection. Several sensing devices used for this class of applications have maximum performance when centered on the axis of the pipe. In order to simulate this scenario a path following control strategy which consists of tracking the center line of the pipe with the head (first module with respect to the forward motion) of the articulated robot is considered. The path following control defines the desired kinematics, and it is coupled to a torque control to generate the torques necessary to track such desired kinematics.

The articulated mobile robot formed with $n$ modules presents $n+2$ degrees of freedom, see Section 2. Here we choose to control the pose of the head of the articulated vehicle, described by the vector $\left(x_{G_{1}}, y_{G_{1}}, \theta_{1}\right)$, along with the orientations $\theta_{2}, \ldots, \theta_{n}$ of the other modules. Therefore the feedback loop in the path following control generates as many inputs $(n+2)$ as the dimension of a minimal set of coordinates that describes the state of the articulated robot. The current choice implies that the center of mass of the modules other than the head do not necessarily track the axis of the pipeline, whereas the local orientation of the path is tracked. Different choices are possible to achieve different objectives, as for example tracking the path with the center of mass of each module, which require the control inputs to depend on the local distances between the centers of mass and the path.

Frenet frames technique is often used in path following problems [26]. A Frenet frame is defined for each module $M_{i}$ by considering the projection of the center of mass $G_{i}$ on the path. Consistently with the parametric description of the walls in Section 3 , let $\Pi(s) \in \mathbb{R}^{2}$ be the path to be tracked, with arclength parameter $s \in \mathbb{R}$. The Frenet frame associated to the curve $\boldsymbol{\Pi}$ consists of the triplet $\{s, \mathbf{T}, \mathbf{N}\}$, with $\mathbf{T}$ and $\mathbf{N}$ being respectively the unit tangent and the unit normal to the curve at $s$. In order to control the pose of the head and the orientation (with respect to the path) of the other modules, we define the error

$$
\begin{array}{r}
\varepsilon_{X}:=X^{d}-X \\
X=\left(x_{G_{1}}, y_{G_{1}}, \theta_{1}, \theta_{2}, \ldots, \theta_{n}\right) \\
X^{d}=\left(\Pi_{x}\left(s_{1}\right), \Pi_{y}\left(s_{1}\right), \theta_{\Pi}\left(s_{1}\right), \ldots \theta_{\Pi}\left(s_{n}\right)\right)
\end{array}
$$


where $\Pi_{x}$ and $\Pi_{y}$ are the global Cartesian components of the point $\Pi, s_{i}$ is the arclength associated to the projection of $G_{i}$ on the path, and $\theta_{\Pi}$ is the global orientation of the tangent $\mathbf{T}$, defined by $\tan \theta_{\Pi}=T_{y} / T_{x}$. Therefore $X$ is a minimal set of coordinates, and $X^{d}$ (that depends on the path) is the corresponding collection of desired states for $X$. In order to asymptotically drive the head of the robot to the path and to track the orientation of the path with the other modules, we define the variable $\bar{X}^{d}$ with first order evolution described by

$$
\dot{\bar{X}}^{d}=\mathbf{K} \varepsilon_{X}+\dot{X}^{d}
$$

where $\mathbf{K}=\operatorname{diag}\left[K_{x}, K_{y}, K_{\theta_{1}}, \ldots, K_{\theta_{n}}\right] \in \mathbb{R}^{n+2 \times n+2}$ is a positive definite gain matrix. The state $\bar{X}^{d}$ can be interpreted as the desired kinematics dictated by the path follower, that is mapped to the desired state $\bar{\xi} d$ through the inverse kinematics. Since $\left\{\cos \theta_{\Pi}\left(s_{1}\right), \sin \theta_{\Pi}\left(s_{1}\right)\right\}$ are the components of the tangent vector $\mathbf{T}\left(s_{1}\right)$, we have

$$
\dot{X}^{d}=V\left(\cos \theta_{\Pi}\left(s_{1}\right), \sin \theta_{\Pi}\left(s_{1}\right), \kappa_{\Pi}\left(s_{1}\right), \ldots, \kappa_{\Pi}\left(s_{n}\right)\right)
$$

with the identifications $\dot{s}_{i}=V$ (forward speed) and $\partial \theta_{\Pi} / \partial s_{i}=\kappa_{\Pi}$ (curvature), consistently with the relation

$$
\dot{\theta}_{\Pi}\left(s_{i}\right)=\dot{s}_{i} \frac{\partial \theta_{\Pi}}{\partial s_{i}}
$$

Therefore if the error $\varepsilon_{X}$ is zero, the desired kinematics corresponds to a forward motion with constant speed $V$, with the head's center of mass on the path, and with the modules oriented parallel to the local tangent (nearest point) of the path.

\subsection{Torque Control}

As sketched in Fig. 6, we control the robot in the Cartesian space which involves the use of inverse geometric and inverse kinematic models. The desired kinematics $\left\{\bar{\xi}^{d}, \dot{\bar{\xi}}^{d}\right\}$ is provided by the path following control loop and by the inverse kinematic model. The joint torques are generated by a PD controller in the form

$$
\boldsymbol{\tau}=\mathbf{K}_{\mathrm{p}} \varepsilon_{\xi}+\mathbf{K}_{\mathrm{d}} \dot{\varepsilon} \xi
$$

with $\mathbf{K}_{\mathrm{p}}$ and $\mathbf{K}_{\mathrm{d}}$ being respectively the proportional and differential gain matrices that are given by

$$
\begin{array}{r}
\mathbf{K}_{\mathrm{p}}=\operatorname{diag}\left[\mathbf{K}_{\mathrm{p} 1}, \ldots, \mathbf{K}_{\mathrm{p} n}\right] \\
\mathbf{K}_{\mathrm{d}}=\operatorname{diag}\left[\mathbf{K}_{\mathrm{d} 1}, \ldots, \mathbf{K}_{\mathrm{d} n}\right]
\end{array}
$$

with $\mathbf{K}_{\mathrm{p} i}=\left[0_{1 \times 7}, K_{\mathrm{p} i}^{s}, K_{\mathrm{p} i}^{s}, K_{\mathrm{p} i}^{w}, K_{\mathrm{p} i}^{w}, 0_{1 \times 4}\right]$ such that $K_{\mathrm{p} i}^{s} \in \mathbb{R}$ and $K_{\mathrm{p} i}^{w} \in \mathbb{R}$ are the proportional gains for controlling the torques of the shoulder joints and the wheels shafts respectively, and $\mathbf{K}_{\mathrm{d} i}=\left[0_{1 \times 7}, K_{\mathrm{d} i}^{s}, K_{\mathrm{d} i}^{s}, K_{\mathrm{d} i}^{w}, K_{\mathrm{d} i}^{w}, 0_{1 \times 4}\right]$ such that $K_{\mathrm{d} i}^{s} \in \mathbb{R}$ and $K_{\mathrm{d} i}^{w} \in \mathbb{R}$ are respectively the derivative gains of the shoulder joints and the wheels shafts torque controllers. $\varepsilon_{\xi}=\bar{\xi}^{d}-\xi$ and $\dot{\varepsilon}_{\xi}=\dot{\bar{\xi}}^{d}-\dot{\xi}$ are the position and velocity error signals with $\bar{\xi}^{d}$ and $\dot{\bar{\xi}}^{d}$ being the desired position and desired velocity vectors. 
The PD controller tracks a time invariant desired kinematics with zero steady-state error. In order to prove it, we first transform the system of equations (51) by introducing the matrix $\mathbf{P} \in \mathbb{R}^{n+2 \times 15 n}$ whose rows are $n+2$ linearly independent vectors in the null space of $\mathbf{C}$. We define the reduced velocity $\dot{\eta} \in \mathbb{R}^{n+2}$ by

$$
\dot{\xi}=\mathbf{P}^{\top} \dot{\eta}
$$

Substitution into equation (51b) gives

$$
\mathbf{C P}^{\top} \dot{\eta}=0
$$

which holds for all $\dot{\eta} \in \mathbb{R}^{n+2}$. Substitution into equation (51a), and pre-multiplication by $\mathbf{P}$ gives the reduced system

$$
\mathbf{M}^{\star}(\eta) \ddot{\eta}+\mathbf{W}(\dot{\eta}, \eta) \dot{\eta}=\tau^{\star}(\eta)
$$

by accounting for the relation $\mathbf{P C}=\mathbf{0}$. Additional symbols in (63) are given by $\mathbb{R}^{n+2 \times n+2} \ni \mathbf{M}^{\star}(\eta)=$ $\mathbf{P M P} \mathbf{P}^{\top}, \mathbb{R}^{n+2 \times n+2} \ni \mathbf{W}(\dot{\eta}, \eta)=\mathbf{P M} \dot{\mathbf{P}}^{\top}$, and $\mathbb{R}^{n+2}(\eta) \ni \boldsymbol{\tau}^{\star}=\mathbf{P} \boldsymbol{\tau}$.

A time invariant path as described in the previous section is defined by constant $\theta_{\Pi}$. Indeed in this case the desired path kinematics is defined by a constant set provided that the forward speed is constant. By adapting a well known result, see for example [40, Chapter 8], we can prove that the system (53a) asymptotically tracks $n+2$ time invariant desired parameters. To do so we consider the Lyapunov function

$$
\mathcal{V}=\frac{1}{2} \dot{\eta}^{\top} \mathbf{M}^{\star} \dot{\eta}+\frac{1}{2} \varepsilon_{\xi}^{\top} \mathbf{K}_{\mathrm{p}} \varepsilon_{\xi}
$$

Time deriving we obtain

$$
\dot{\mathcal{V}}=\dot{\eta}^{\top} \mathbf{M}^{\star} \ddot{\eta}+\frac{1}{2} \dot{\eta}^{\top} \dot{\mathbf{M}}^{\star} \dot{\eta}+\varepsilon_{\xi}^{\top} \mathbf{K}_{\mathrm{p}} \dot{\varepsilon}_{\xi}
$$

For a time invariant desired trajectory, that is $\dot{\xi}^{d}=0$, substitution from (63) and (61) gives

$$
\dot{\mathcal{V}}=\frac{1}{2} \dot{\eta}^{\top}\left(\dot{\mathbf{M}}^{\star}-2 \mathbf{W}\right) \dot{\eta}+\dot{\eta}^{\top} \boldsymbol{\tau}^{\star}-\dot{\eta}^{\top} \mathbf{P} \mathbf{K}_{\mathrm{p}} \varepsilon_{\xi}
$$

By adopting the linear feedback $\tau^{\star}=\mathbf{P} \boldsymbol{\tau}=\mathbf{P} \mathbf{K}_{\mathrm{p}} \varepsilon_{\xi}+\mathbf{P} \mathbf{K}_{\mathrm{d}} \dot{\varepsilon}_{\xi}$ with time invariant desired trajectory, and by the fact that the matrix $\dot{\mathbf{M}}^{\star}-2 \mathbf{W}$ is skew-symmetric since

$$
\dot{\mathbf{M}}^{\star}-2 \mathbf{W}=\dot{\mathbf{P}} \mathbf{M} \mathbf{P}^{\top}-\mathbf{P M} \dot{\mathbf{P}}^{\top}
$$

the time derivative of the Lyapunov function simplifies to

$$
\dot{\mathcal{V}}=-\dot{\eta}^{\top} \mathbf{K}_{\mathrm{d}}^{\star} \dot{\eta} \leq 0
$$


where $\mathbf{K}_{\mathrm{d}}^{\star}=\mathbf{P} \mathbf{K}_{\mathrm{d}} \mathbf{P}^{\top}$, and the equality holds for $\dot{\eta}=0$. Therefore $\dot{\mathcal{V}}$ decreases along the trajectories for nonzero $\dot{\eta}$. In order to complete the proof we need to show that the largest invariant set is $\varepsilon_{\xi}=0$. To do so, assume that $\dot{\mathcal{V}} \equiv 0$ (which means that $\dot{\mathcal{V}}$ is identically equal to zero). Then (68) implies $\dot{\eta} \equiv 0$ and therefore $\ddot{\eta} \equiv 0$. The equation of motion (22) with linear feedback then implies $\mathbf{K}_{\mathrm{p}} \varepsilon_{\xi}=0$. Let

$$
\delta:=\left(\delta_{1}, \ldots, \delta_{n}\right)
$$

with

$$
\delta_{i}:=\left(\alpha_{i}^{r}, \alpha_{i}^{l}, \theta_{i}^{r}, \theta_{i}^{l}\right)
$$

be the collection of actuated states, corresponding to nonzero entries of the diagonal gain matrix $\mathbf{K}_{\mathrm{p}}$. Consider the map

$$
\boldsymbol{\varphi}: \mathbb{R}^{n+2} \ni \eta \mapsto \boldsymbol{\varphi}(\eta)=\delta \in \mathbb{R}^{4 n}
$$

By indicating with $\overline{\mathbf{K}}_{\mathrm{p}}$ the diagonal matrix obtained from $\mathbf{K}_{\mathrm{p}}$ by extracting the nonzero elements, we have

$$
\mathbf{K}_{\mathrm{p}} \varepsilon_{\xi}=\overline{\mathbf{K}}_{\mathrm{p}} \varepsilon_{\delta}
$$

where $\varepsilon_{\delta}=\delta-\delta^{d}$, with $\delta^{d}$ being the desired state for $\delta$. Since $\overline{\mathbf{K}}_{\mathrm{p}}$ is full rank

$$
\overline{\mathbf{K}}_{\mathrm{p}} \varepsilon_{\delta}=\mathbf{0} \Rightarrow \varepsilon_{\delta}=0
$$

By LaSalle's theorem [48, Chapter 5] the equilibrium is globally asymptotically stable, and therefore $\delta \rightarrow \delta^{d}$. In terms of the minimal set $\eta$ this implies that $\boldsymbol{\varphi}(\eta) \rightarrow \boldsymbol{\varphi}\left(\eta^{d}\right)$. If the map $\boldsymbol{\varphi}$ is one to one, then $\varepsilon_{\eta}=\eta^{d}-\eta$ is globally asymptotically stable.

Moreover, if the map $\varphi$ is non-singular in a neighborhood of the desired state $\eta^{d}$, it is well defined the linearization

$$
\delta=\delta^{d}+\boldsymbol{\Phi}\left(\eta-\eta^{d}\right)
$$

where $\delta^{d}=\boldsymbol{\varphi}\left(\eta^{d}\right)$, and $\boldsymbol{\Phi}=\nabla_{\eta} \boldsymbol{\varphi}\left(\eta^{d}\right) \in \mathbb{R}^{4 n \times n+2}$ is the gradient of $\boldsymbol{\varphi}$ at $\eta^{d}$. In this case the equilibrium $\varepsilon_{\eta}$ is locally asymptotically stable since $\varepsilon_{\delta}=0 \Rightarrow \boldsymbol{\Phi} \varepsilon_{\eta}=0$.

Note that for $n>0$ the number of actuated states (4n) always exceeds the number of degrees of freedom of the articulated robot $(n+2)$, and therefore the mechanism is redundantly actuated. Based on the discussion above, it is clear that if the Jacobian $\mathbf{C}$ is full rank the actuated states collected in $\delta$ asymptotically track the corresponding desired ones. On the neighborhood of a non-singular desired minimal configuration $\eta^{d}$, the inverse relation between $\eta$ and $\delta$ can be expressed as

$$
\eta=\eta^{d}+\boldsymbol{\Phi}^{\dagger} \varepsilon_{\delta}+\left(\mathbf{I}_{n+2}-\boldsymbol{\Phi}^{\dagger} \boldsymbol{\Phi}\right) \bar{\eta}
$$


where $\boldsymbol{\Phi}^{\dagger}=\boldsymbol{\Phi}^{\top}\left(\boldsymbol{\Phi} \boldsymbol{\Phi}^{\top}\right)^{-1} \in \mathbb{R}^{n+2 \times 4 n}$ is the right Moore-Penrose pseudoinverse of $\boldsymbol{\Phi}$, and $\left(\mathbf{I}_{n+2}-\right.$ $\left.\boldsymbol{\Phi}^{\dagger} \boldsymbol{\Phi}\right) \bar{\eta}$ is a vector in the null space of $\boldsymbol{\Phi}$ for every $\bar{\eta} \in \mathbb{R}^{n+2}$. The redundancy allows to exploit the arbitrariness of $\bar{\eta}$ to achieve different desired states for $\eta$ for the same $\varepsilon_{\delta}$ (eventually zero, as in our case). However, here we consider the minimum least square solution of the inverse kinematics and set $\bar{\eta}=0$.

\subsubsection{Crossing a Pipe with Changing Diameter}

The objective of this simulation is to illustrate the model of the multibody mechanism by showing the performance of the control strategy in following the center line of the pipe. In addition, it shows how the structure adapts to changes in the pipe diameter (a restriction followed by an enlargement). The first part of the pipe is $2.5 \mathrm{~m}$ long with diameter $42 \mathrm{~cm}$ followed by a $1 \mathrm{~m}$ length pipe with diameter of $34 \mathrm{~cm}$ and a final portion $2.5 \mathrm{~m}$ long with diameter of $42 \mathrm{~cm}$. The geometry of each module is determined by the set of parameters $h=35 \mathrm{~cm}$ (height), $W=10 \mathrm{~cm}$ (width), $\rho=3 \mathrm{~cm}$ (wheels radius), $L=24 \mathrm{~cm}$ (arm length), and $\gamma=0.5$. The kinematic path following is defined by the the gains $K_{x}=K_{y}=3$ and $K_{\theta}=3$. The discrete time simulation scheme is run with sampling time $0.025 \mathrm{~s}$. The gains for the torque control are $K_{\mathrm{p} i}^{s}=0.7, K_{\mathrm{d} i}^{s}=0.2$, and $K_{\mathrm{d} i}^{w}=0.03$ for $i=1, \ldots, n$. The proportional part of the wheel torque control is set to zero since the angular position of the wheel shaft is unbounded.

Figure 7 depicts a snapshot of three positions of the vehicle inside the pipe: initial, mid-length and final. The distance and orientation errors are stabilized asymptotically as shown in Figure 8, and as qualitatively confirmed by the inspection of Figure 7. Since the system is centered on the axis of the pipeline by the time the first module reaches the restriction, the torques generated by the controller to react to the restriction are of equal magnitude and opposite sign, as shown in Table 1 for the head of the robot. Tables reporting results for the other modules are given in the Appendix. Due to this symmetry, the net action on the module due to the restriction is zero and therefore there is no perturbation. Same considerations hold for the enlargement of the pipe.

For the first two modules, the joint torques corresponding to this simulation are displayed on Figure 9. For all the modules, the torque curves exhibit a similar shape, that is flat with two peak areas that propagate among the modules. These peaks are the joints reaction to the two successive changes in pipe diameter. Since the articulated mechanism is centered on the axis by the time the first module reaches the restriction, the torques generated are the same for each module with a characteristic time shift that depends on the distance among wheels belonging to contiguous modules, and on the updating time step. If we consider the restriction as an obstacle, as the wheels meet the pipe restriction they decelerate while the arms are closing. When they leave the restriction and enter the enlargement, the wheels accelerate while the arms spread. Note that the right wheel angular velocity is anti-clockwise when a module moves in the direction of increasing arc length, which means that a negative value in torque for the right wheel shaft corresponds to an acceleration in the module's direction of motion. This coordinated motion is due to the enforcement of the internal constraints (closed kinematic chain) and external constraints (permanent contact of the wheels with the pipe) while the module tracks the center line with a constant velocity. The rest of the time the torques are almost zero due to the fact that the pipe portions in which the vehicle is travelling are straight. However, one can notice slight disturbances in the wheel shaft torque curves of every module due to the manoeuvres of other modules each time they cross the restriction.

\subsubsection{Crossing a Pipe with an Elbow}

Here, we show the operation of the robot while crossing two straight pipe sections connected by a $3 \pi / 4$ elbow. The diameter of the pipe is constant but the pipe presents a change in curvature. We follow a path with piecewise constant curvature. A constant curvature allows for the forward and lateral 
motions to be decoupled, so that the path following problem can be treated as the problem of determining the asymptotic stability of the lateral motion [49], with the system driven by the longitudinal motion. Numerical values for the gains of the path following controller and for the torque controller are the same as the ones listed above for the case of straight pipe with restriction, and the sampling time for the discrete time integration algorithm is $0.02 \mathrm{~s}$ for simulations involving one module, and $0.025 \mathrm{~s}$ for simulations involving the articulated vehicle.

For a single module, Figure 10 illustrates three key positions while crossing the pipe with elbow. The distance and orientation errors for this simulation depicted on Figure 11 converge to zero with two saddle points that are due to the two successive changes in curvature when the module enters and leaves the elbow. This emerges also on the curves depicted on Figure 12, showing the reaction of the joints in terms of torque in response to the curvature changes.

For the case of the articulated vehicle, we apply the same control scheme as for the pipe with restriction, with the same numerical values of the geometric and control parameters. Figure 13 shows a snapshot of the articulated vehicle inside the pipe with elbow. Distance and orientation error curves are shown on Figures 14. Since the head pose is fully controlled its distance and orientation errors exhibit the expected better result, especially in the distance error, as compared to the the rest of modules for which only the orientations are controlled. The torques applied by the joints are shown in Figure 15 for the four modules. Notice the difference in the efforts produced by the joints of the modules as part of the articulated vehicle when compared to the case of a module evolving separately (Figure 12). Every module is subjected to inner structural constraints and also to hitching constraints. In the case of a change of curvature, the interactions between the modules forming the articulated vehicle are important and visible compared to the case of crossing a straight pipe with changing diameter. This shows that a change of curvature introduces a challenging scenario as the proportional controller cannot compensate for the disturbance introduced by it, as opposed to the case of change of diameter of the cross section.

The rolling without slipping constraint adopted here is enforced by virtue of a set of Lagrange multipliers that quantify the contact forces (Cartesian components) required to guarantee that such constraint is not violated. This does not imply that the contact forces are physically admissible, as for example the friction required to enforce the no slip condition could be too high for the materials involved. The assessment of the physical compatibility would require the introduction of a specific set of constitutive relations that relate the contact forces to the kinematic duals (tangential velocities), as it is done for example in [13]. However, within the model considered in this work, the contact forces involved in the evolution of the robot when the rolling without slipping constraints hold, are computed directly through equation (53b), and they can be used to estimate the material properties (coefficients of friction) necessary to guarantee the non violation of the constraint, or eventually the non compatibility with commonly used materials. To illustrate this we show in Figure 16 the coefficients of friction, obtained through a simple Coulomb friction model, as the ratio

$$
\mu=\frac{F_{T}}{F_{N}}
$$

between the tangential and the normal components, $F_{T}$ and $F_{N}$ respectively, of the contact forces between walls and wheels. Results refer to the example of one module crossing an elbow, and the tangential and normal components are obtained from the Cartesian components $\lambda_{x}$ and $\lambda_{y}$ by solving the linear system

$$
\begin{aligned}
& \lambda_{x}=F_{T} T_{x}+F_{N} N_{x} \\
& \lambda_{y}=F_{T} T_{y}+F_{N} N_{y}
\end{aligned}
$$


where $T_{x}, T_{y}, N_{x}$, and $N_{y}$ are the Cartesian components of the tangent and of the normal vector fields.

The coefficients of friction in Figure 16 are shown for the right and for the left wheels, and therefore two sets of Lagrange multipliers are involved, with corresponding two Frenet frames (and unit vectors) associated to the two walls. Peaks of the values of the estimated coefficients of friction may correspond to non-realizable scenarios in terms of materials, as typically the coefficients of friction for common engineering pairs of materials range between 0.1 to 1.5 .

The gap between the wheels nearest points to the wall and the corresponding point on the wall, plotted in Figure 17, shows that the rolling without slipping constraints are correctly enforced during the simulation.

\section{Conclusions}

In this paper, we derived the dynamics of an articulated mobile robot tailored for the exploration of confined environments, more specifically for in-pipe inspection applications. The kinematics of the robot is described by a chain of parallel mechanisms on mobile platforms. This kinematics is characterized by a larger number of degrees of freedom, and therefore available control inputs, with respect to other articulated vehicles such as the $n$-trailer. The augmented number of degrees of freedom corresponds to more versatility in executing some pipe inspections maneuvers such as turning sharp angles while maintaining the robot axially centered to maximize sensor performance.

The model derivation is based on Lagrange's formulation for constrained multibody dynamics. We first derived the equations of motion of one module that we extended to the whole articulated vehicle. The model is illustrated through simulations of motion inside a pipe. A path following controller is used together with a torque controller for the navigation of the vehicle to track the center line of a pipe for two different operational scenarios: a straight pipe with changing diameter and a pipe with $3 \pi / 4$ elbow. Simulation results illustrate the effectiveness of the dynamic model in predicting the actuation efforts involved in the two considered operational scenarios, that can be typically encountered when there is an obstruction of the cross section of the pipe, and when the robot has to negotiate a change of direction. The model allows to simulate critical situations and to predict the actions necessary to track a desired trajectory for sensor performance maximization. It is therefore possible to design control gains that minimize the energy consumption associated to control actions, and ultimately to maximize the autonomy of the robot.

The design of advanced control laws to improve the coordination of the modules and to handle the more general case of paths with varying curvatures is part of ongoing works. The model of the three dimensional version of the robot is also part of future work.

\section{Acknowledgements}

This work was partially funded by the Federal Economic Development Agency for Southern Ontario through the SME4SME program, and partially by NSERC through the Discovery program.

A special acknowledgement to InvoDane Engineering, Ltd. for the collaboration through the grant SME4SME. 


\section{References}

[1] Hirose, S., and Morishima, A., 1990. "Design and control of a mobile robot with an articulated body". Journal of Robotics Research, 9(2), pp. 99-114.

[2] Mirats Tur, J., and Garthwaite, W., 2010. "Robotic devices for water main in-pipe inspection: A survey". Journal of Field Robotics, 27(4), pp. 491-508.

[3] Mazumdar, A., and Asada, H. H., 2010. "An Underactuated, Magnetic-Foot Robot for Steel Bridge Inspection". Journal of Mechanics and Robotics-Transactions of the ASME, 2(3), Aug, pp. 031007-031007-9.

[4] Suzumori, K., Wakimoto, S., and Takata, M., 2003. "A miniature inspection robot negotiating pipes of widely varying diameter". In 2003 IEEE International Conference on Robotics and Automation, VOLS 1-3, Proceedings, pp. 2735-2740.

[5] Schempf, H., Mutschler, E., Goltsberg, V., Skoptsov, G., Gavaert, A., and Vradis, G., 2003. "Explorer: Untethered real-time gas main assessment robot system". In Proc. of Int. Workshop on Advances in Service Robotics, ASER'03.

[6] Jamoussi, A., 2005. "Robotic NDE: A new solution for in-line pipe inspection". In Middle East Nondestructive Testing Conference and Exhibition.

[7] Fjerdingen, S. A., Liljebäck, P., and Transeth, A. A., 2009. "A snake-like robot for internal inspection of complex pipe structures (PIKo)”. In 2009 IEEE/RSJ International Conference on Intelligent Robots and Systems, pp. 5665-5671.

[8] Shin, H., Jeong, K.-M., and Kwon, J.-J., 2010. "Development of a Snake Robot Moving in a Small Diameter Pipe". In International Conference on Control, Automation and Systems (ICCAS 2010), pp. 1826-1829.

[9] Dertien, E., Stramigioli, S., and Pulles, K., 2011. "Development of an inspection robot for small diameter gas distribution mains". In Robotics and Automation (ICRA), 2011 IEEE International Conference on, pp. 5044-5049.

[10] Transeth, A. A., and Pettersen, K. Y., 2006. "Developments in snake robot modeling and locomotion”. In International Conference on Control, Automation, Robotics and Vision ICARCV, pp. 1-8.

[11] Wiriyacharoensunthorn, P., and Laowattana, S., 2002. "Analysis and design of a multi-link mobile robot (serpentine)". In IEEE International Conference on Industrial Technology, Vol. 2, pp. 694699.

[12] Ostrowski, J., and Burdick, J., 1996. "Gait kinematics for a serpentine robot". In Proceedings of the IEEE International Conference on Robotics and Automation, Vol. 2, pp. 1294-1299.

[13] Transeth, A. A., Leine, R. I., Glocker, C., Pettersen, K. Y., and Liljebäck, P., 2008. "Snake robot obstacle-aided locomotion: modeling, simulations, and experiments". IEEE Transactions on Robotics, 24(1), pp. 88-104.

[14] Ma, S., and Tadokoro, N., 2006. "Analysis of creeping locomotion of a snake-like robot on a slope”. Autonomous Robots, 20(1), pp. 15-23.

[15] Bayraktaroglu, Z. Y., and Blazevic, P., 2005. "Understanding snakelike locomotion through a novel push-point approach”. Journal of dynamic systems, measurement, and control, 127(1), pp. 146152.

[16] Hopkins, J. K., Spranklin, B. W., and Gupta, S. K., 2011. "A Case Study in Optimization of Gait and Physical Parameters for a Snake-Inspired Robot Based on a Rectilinear Gait". Journal of Mechanics and Robotics-Transactions of the ASME, 3(1), Feb.

[17] Briot, S., Glazunov, V., and Arakelian, V., 2013. "Investigation on the Effort Transmission in Planar Parallel Manipulators". Journal of Mechanics and Robotics-Transactions of the ASME, 5(1), Feb.

[18] Carretero, J. A., Ebrahimi, I., and Boudreau, R., 2012. “Overall Motion Planning for Kinematically 
Redundant Parallel Manipulators". Journal of Mechanics and Robotics-Transactions of the ASME, 4(2), May.

[19] Hu, B., Yu, J., Lu, Y., Sui, C., and Han, J., 2012. "Statics and Stiffness Model of Serial-Parallel Manipulator Formed by $k$ Parallel Manipulators Connected in Series". Journal of Mechanics and Robotics-Transactions of the ASME, 4(2), May.

[20] Bolzern, P., DeSantis, R., Locatelli, A., and Togno, S., 1996. "Dynamic model of a two-trailer articulated vehicle subject to nonholonomic constraints". Robotica, 14(4), pp. 445-450.

[21] Ute, J., and Ono, K., 2002. "Fast and efficient locomotion of a snake robot based on self-excitation principle". In 7TH International Workshop on Advanced Motion Control, Proceedings, pp. 532539.

[22] Transeth, A. A., Leine, R. I., Glocker, C., and Pettersen, K. Y., 2008. “3-D snake robot motion: Nonsmooth modeling, simulations, and experiments". IEEE Transactions on Robotics, 24(2), pp. 361-376.

[23] Transeth, A. A., Leine, R. I., Glocker, C., Pettersen, K. Y., and Liljebäck, P., 2008. "Snake robot obstacle-aided locomotion: Modeling, simulations, and experiments". IEEE Transactions on Robotics, 24(1), pp. 88-104.

[24] Liljebäck, P., Pettersen, K. Y., Stavdahl, O., and Gravdahl, J. T., 2010. “A simplified model of planar snake robot locomotion”. In IEEE/RSJ 2010 International Conference on Intelligent Robots and Systems (IROS 2010), pp. 2868-2875.

[25] Liljebäck, P., Pettersen, K., Stavdahl, O., and Gravdahl, J., 2010. "Hybrid modelling and control of obstacle-aided snake robot locomotion". IEEE Transactions on Robotics, 26(5), pp. 781-799.

[26] Murugendran, B., Transeth, A. A., and Fjerdingen, S. A., 2009. "Modeling and Path-following for a Snake Robot with Active Wheels". In 2009 IEEE-RSJ International Conference on Intelligent Robots and Systems, pp. 3643-3650.

[27] Li, N., Zhao, T., and Zhao, Y., 2008. "The dynamic modeling of snake-like robot by using nominal mechanism method". In ICIRA '08 Proceedings of the First International Conference on Intelligent Robotics and Applications: Part I, pp. 1185-1194.

[28] Liljebäck, P., Pettersen, K., Stavdahl, O., and Gravdahl, J., 2012. “A review on modelling, implementation, and control of snake robots". Robotics and Autonomous Systems, 60(1), pp. 29-40.

[29] Samin, J.-C., and Fisette, P., 2003. Symbolic Modeling of Multibody Systems. Kluwer Academic Pub.

[30] Bendtsen, C., and Thomsen, P., 1999. Numerical Solution of Differential Algebraic Equations. IMM, Department of Mathematical Modeling, Technical University of Denmark.

[31] Wijckmans, P., 1996. "Conditionning of differential algebraic equations and numerical solution of multibody dynamics". PhD thesis, Technische Universiteit Eindhoven.

[32] Baumgarte, J., 1972. "Stabilization of constraints and integrals of motion in dynamical systems". Computer Methods in Applied Mechanics and Engineering, 1(1), pp. 1-16.

[33] Cline, M. B., and Pai, D. K., 2003. "Post-stabilization for rigid body simulation with contact and constraints". In 2003 IEEE International Conference on Robotics and Automation, Proceedings, Vol. 1-3, pp. 3744-3751.

[34] Ascher, U., Chin, H., Petzold, L., and Reich, S., 1995. "Stabilization of constrained mechanical systems with DAEs and invariant manifolds". Mechanics of Structures and Machines, 23, pp. 135157.

[35] Bauchau, O., and Laulusa, A., 2008. "Review of contemporary approaches for constraint enforcement in multibody systems". Journal of Computational and Nonlinear Dynamics, 3(1), pp. 1-8.

[36] Sarfraz, H., Spinello, D., Gueaieb, W., and Douadi, L., 2013. "Critical maneuvers of an autonomous parallel robot in a confined environment". In Proceedings of the International Conference of Mechanical Engineering and Mechatronics (ICMEM), pp. 196-1. 
[37] Gosselin, C., and Angeles, J., 1990. "Singularity analysis of closed-loop kinematic chains". IEEE Transactions on Robotics and Automation, 6(3), pp. 281-290.

[38] Merlet, J. P., 2006. Parallel Robots. Springer.

[39] Bernstein, D. S., 2011. Matrix Mathematics: Theory, Facts, and Formulas (Second Edition). Princeton University Press.

[40] Spong, M. W., Hutchinson, S., and Vidyasagar, M., 2006. Robot Modeling and Control. Wiley.

[41] Flannery, M., 2004. "The enigma of nonholonomic constraints". American Journal of Physics, 73(3), pp. 265-272.

[42] Hemami, H., and Weimer, F. C., 1981. "Modeling of nonholonomic dynamic systems with applications". Journal of Applied Mechanics, 48(1), pp. 177-182.

[43] Metiku, R., 2004. “Computer-aided dynamic force analysis of four-bar planar mechanism.”. Master's thesis, Addis Ababa University. School of Graduate Studies.

[44] Poursina, M., and Anderson, K. S., 2013. "An extended divide-and-conquer algorithm for a generalized class of multibody constraints". Multibody System Dynamics, 29(3), pp. 235-254.

[45] Kreutz-Delgado, K., Jain, A., and Rodriguez, G., 1991. "Recursive formulation of operational space control". In Robotics and Automation, 1991. Proceedings., 1991 IEEE International Conference on, pp. 1750-1753.

[46] Poursina, M., and Anderson, K. S., 2013. "Canonical ensemble simulation of biopolymers using a coarse-grained articulated generalized divide-and-conquer scheme”. Computer Physics Communications, 184(3), pp. 652-660.

[47] Yu, Q., and Chen, I.-M., 2000. "A direct violation correction method in numerical simulation of constrained multibody systems". Computational Mechanics, 26(1), pp. 52-57.

[48] Sastry, S. S., 1999. Nonlinear Systems: Analysis, Stability, and Control. Springer.

[49] Altafini, C., 2002. "Following a path of varying curvature as an output regulation problem". IEEE Transactions on Automatic Control, 47(9), pp. 1551-1556.

\section{Appendix A: Complement to the section on kinematics}

State dependent coefficients appearing on the closure kinematic equations (22)

$$
\begin{array}{r}
a_{i}^{r}=\frac{1}{2} W \cos \theta_{i}+L \sin \left(\theta_{i}+\alpha_{i}^{r}\right)-H \sin \theta_{i} \\
c_{i}^{r}=-L \sin \left(\theta_{i}+\alpha_{i}^{r}\right) \\
b_{i}^{r}=\frac{1}{2} W \sin \theta_{i}-L \cos \left(\theta_{i}+\alpha_{i}^{r}\right)+H \cos \theta_{i} \\
d_{i}^{r}=L \cos \left(\theta_{i}+\alpha_{i}^{r}\right) \\
a_{i}^{l}=-\frac{1}{2} W \cos \theta_{i}-L \sin \left(\theta_{i}+\alpha_{i}^{l}\right)-H \sin \theta_{i} \\
c_{i}^{l}=L \sin \left(\theta_{i}+\alpha_{i}^{l}\right) \\
b_{i}^{l}=-\frac{1}{2} W \sin \theta_{i}+L \cos \left(\theta_{i}+\alpha_{i}^{l}\right)+H \cos \theta_{i} \\
d_{i}^{l}=-L \cos \left(\theta_{i}+\alpha_{i}^{l}\right)
\end{array}
$$


Parallel and serial Jacobians in (32)

$$
\mathbf{J}_{p_{i}}=\left[\begin{array}{c:c}
\mathbf{I}_{2} & a_{i}^{r} \\
\hdashline \mathbf{I}_{2} & b_{i}^{r} \\
\hdashline 0_{4 \times 2} & a_{i}^{l} \\
\hdashline b_{i}^{l} & 0_{4 \times 1} \\
\hdashline 0_{4 \times 2} & \frac{L}{2} \sin \left(\theta_{i}+\alpha_{i}^{r}\right) \\
& -\frac{L}{2} \cos \left(\theta_{i}+\alpha_{i}^{r}\right) \\
& \frac{L}{2} \sin \left(\theta_{i}+\alpha_{i}^{l}\right) \\
\hdashline & \cos \left(\theta_{i}+\alpha_{i}^{l}\right)
\end{array}\right]
$$

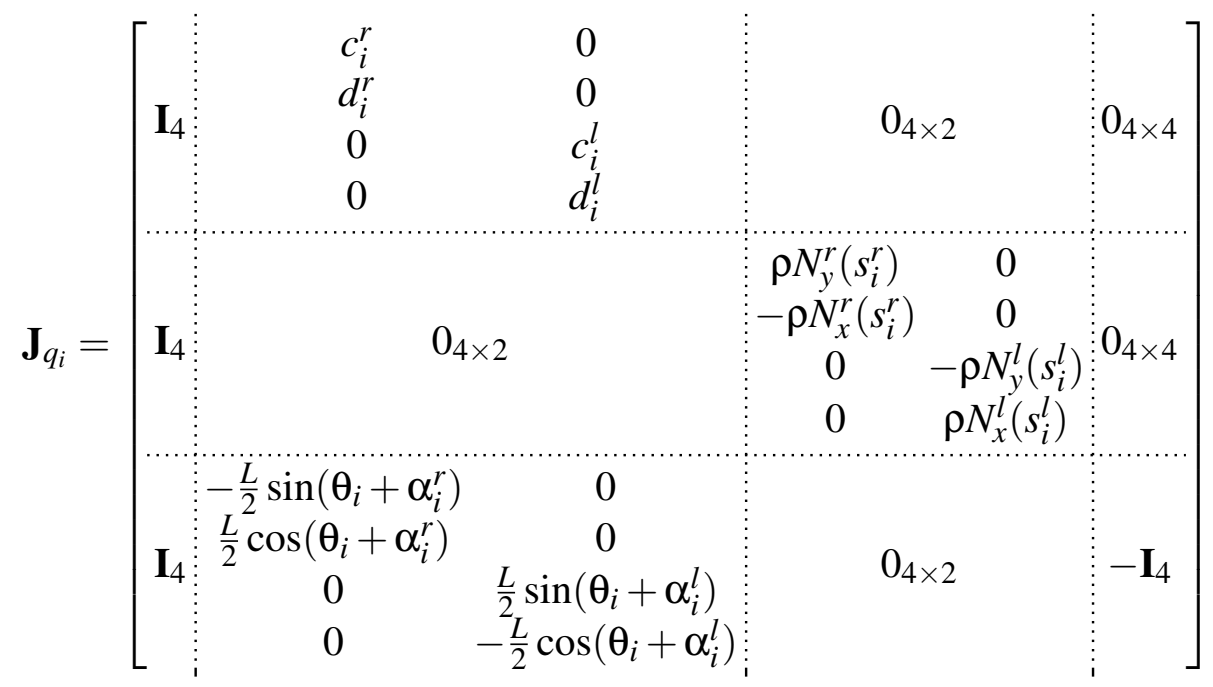

Jacobians in (33)

$$
\mathbf{J}_{p}=\left[\begin{array}{lllllll}
\mathbf{J}_{p_{1}} & & & & & & \\
\mathbf{Q}_{1} & \mathbf{S}_{2} & & & & \\
& \mathbf{J}_{p_{2}} & & & & \\
& \mathbf{Q}_{2} & \mathbf{S}_{3} & & & & \\
& & & & \\
& & \mathbf{J}_{p_{3}} & & & & \\
& & \mathbf{Q}_{3} & \mathbf{S}_{4} & & & \\
& & & \ddots & & \\
& & & & \mathbf{Q}_{n-1} & \mathbf{S}_{n} \\
& & & & & \mathbf{J}_{p_{n}}
\end{array}\right]
$$

$$
\mathbf{Q}_{i}=\left[\begin{array}{ccc}
1 & 0 & L_{b} \sin \theta_{i} \\
0 & 1 & -L_{b} \cos \theta_{i}
\end{array}\right]
$$

$$
\mathbf{S}_{i}=\left[\begin{array}{ccc}
-1 & 0 & L_{f} \sin \theta_{i} \\
0 & -1 & -L_{f} \cos \theta_{i}
\end{array}\right]
$$




$$
\mathbf{J}_{q}=\left[\begin{array}{cccc}
\mathbf{J}_{q_{1}} & & & \\
0_{2 \times n} & & & \\
& \mathbf{J}_{q_{2}} & & \mathbf{0} \\
& 0_{2 \times n} & & \\
\mathbf{0} & & \ddots & \\
& & & \mathbf{J}_{q_{n}}
\end{array}\right]
$$

\section{Appendix B: Additional Numerical Results in Section 5.2.1}

Torques generated by the controller to react to the changes of cross section of the pipeline: results for Modules 2, 3, and 4 are shown respectively in Tables 2, 3, and 4. 


\section{List of Tables}

1 Torque peak values for Module 1 corresponding to the change of cross section of the pipe. 27

2 Torque peak values for Module 2 corresponding to the change of cross section of the pipe. 28

3 Torque peak values for Module 3 corresponding to the change of cross section of the pipe. 29

4 Torque peak values for Module 4 corresponding to the change of cross section of the pipe. 30

\section{List of Figures}

1 Kinematic scheme of the articulated mobile robot. . . . . . . . . . . . . . . . 31

2 Schematic of one module with notation. . . . . . . . . . . . . . . . . 32

3 Right wheel contact with the pipe wall . . . . . . . . . . . . . . 33

4 Two serial singularities: (a) both arms are normal to the pipe walls, (b) only one arm is normal to the wall . . . . . . . . . . . . . . . . . . . 34

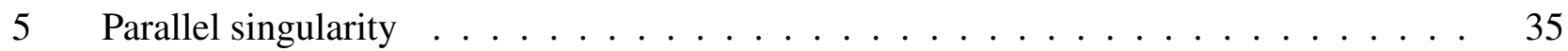

$6 \quad$ PD control scheme. "IK" is the acronym of Inverse Kinematics. . . . . . . . . . . . . 36

7 Key positions in a straight pipe with changing diameter . . . . . . . . . . . . . 37

8 Linear and angular tracking errors when crossing the straight pipe with changing diameter. $M_{1}, \ldots, M_{4}$ are labels for the modules, assigned progressively from the head

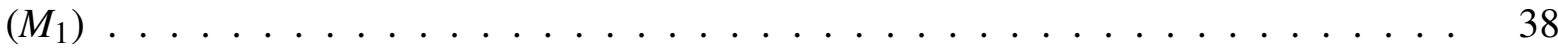

9 Modules' joint torques: crossing the straight pipe . . . . . . . . . . . . . . . . 39

10 Initial, intermediate and final positions of a module when crossing a pipe with elbow, while tracking the axis of the pipe . . . . . . . . . . . . . . . 40

11 Linear and angular tracking errors of a module while tracking the axis of a pipe with elbow. $M_{1}$ labels the only module simulated in this case . . . . . . . . . . . . 41

12 Joint torques of a module when crossing a pipe with elbow and tracking its axis . . . 42

13 Three configurations of the articulated vehicle while tracking the axis of a pipe with elbow 43

14 Linear and angular tracking errors for the articulated vehicle while tracking the axis of a pipe with elbow. $M_{1}, \ldots, M_{4}$ are labels for the modules, assigned progressively from

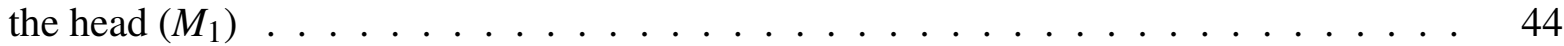

15 Modules' joint torques: crossing a pipe with elbow . . . . . . . . . . . . . . . 45

16 For one module crossing an elbow: coefficients of friction computed by using the Coulomb friction model, with values computed from Lagrange multipliers. . . . . . . 46

17 For one module crossing an elbow: gap between the wall and the wheels. . . . . . . . 47 


\begin{tabular}{l|cc|cc} 
& \multicolumn{2}{|c|}{ Restriction } & \multicolumn{2}{c}{ Enlargement } \\
\hline Time $[\mathrm{s}]$ & 2.800 & 2.975 & 5.025 & 5.125 \\
\hline Torque on right shoulder $[\mathrm{N} \mathrm{m}]$ & -0.1455 & 0.1502 & 0.2563 & -0.3131 \\
Torque on left shoulder $[\mathrm{N} \mathrm{m}]$ & 0.1456 & -0.1502 & -0.2563 & 0.3131 \\
\hline Torque on right wheel shaft $[\mathrm{N} \mathrm{m}]$ & 0.0728 & -0.0312 & -0.2718 & 0.4360 \\
Torque on left wheel shaft $[\mathrm{N} \mathrm{m}]$ & -0.0728 & 0.0312 & 0.2718 & -0.4360 \\
\hline
\end{tabular}

Table 1. Torque peak values for Module 1 corresponding to the change of cross section of the pipe. 


\begin{tabular}{l|cc|cc} 
& \multicolumn{2}{|c|}{ Restriction } & \multicolumn{2}{c}{ Enlargement joint } \\
\hline Time $[\mathrm{s}]$ & 3.500 & 3.7 & 5.725 & 5.825 \\
\hline Torque on right shoulder $[\mathrm{N} \mathrm{m}]$ & -0.1456 & 0.1507 & 0.2554 & -0.3130 \\
Torque on left shoulder $[\mathrm{N} \mathrm{m}]$ & 0.1456 & -0.1507 & -0.2554 & 0.3129 \\
\hline Torque on right shaft $[\mathrm{N} \mathrm{m}]$ & 0.0734 & -0.0297 & -0.2710 & 0.4315 \\
Torque on left shaft $[\mathrm{N} \mathrm{m}]$ & -0.0734 & 0.0297 & 0.2710 & -0.4315 \\
\hline
\end{tabular}

Table 2. Torque peak values for Module 2 corresponding to the change of cross section of the pipe. 


\begin{tabular}{l|cc|cc} 
& \multicolumn{2}{|c|}{ restriction joint } & \multicolumn{2}{c}{ enlargement joint } \\
\hline Time [s] & 4.200 & 4.400 & 6.425 & 6.525 \\
\hline Torque on right shoulder $[\mathrm{N} \mathrm{m}]$ & -0.1456 & 0.1507 & 0.2554 & -0.3130 \\
Torque on left shoulder [N m] & 0.1456 & -0.1507 & -0.2554 & 0.3130 \\
\hline Torque on right wheel shaft $[\mathrm{N} \mathrm{m}]$ & 0.0742 & -0.0298 & -0.2711 & 0.4316 \\
Torque on left wheel shaft $[\mathrm{N} \mathrm{m}]$ & -0.0742 & 0.0298 & 0.2711 & -0.4316 \\
\hline
\end{tabular}

Table 3. Torque peak values for Module 3 corresponding to the change of cross section of the pipe. 


\begin{tabular}{l|cc|cc} 
& \multicolumn{2}{|c|}{ restriction joint } & \multicolumn{2}{c}{ enlargement joint } \\
\hline Time $[\mathrm{s}]$ & 4.925 & 5.100 & 7.125 & 7.225 \\
\hline Torque on right shoulder $[\mathrm{N} \mathrm{m}]$ & -0.1454 & 0.1530 & 0.2555 & -0.3132 \\
Torque on right shoulder $[\mathrm{N} \mathrm{m}]$ & 0.1454 & -0.1530 & -0.2555 & 0.3132 \\
\hline Torque on right shoulder $[\mathrm{N} \mathrm{m}]$ & 0.0684 & -0.0219 & -0.2711 & 0.4322 \\
Torque on right shoulder $[\mathrm{N} \mathrm{m}]$ & -0.0684 & 0.0219 & 0.2711 & -0.4322 \\
\hline
\end{tabular}

Table 4. Torque peak values for Module 4 corresponding to the change of cross section of the pipe. 


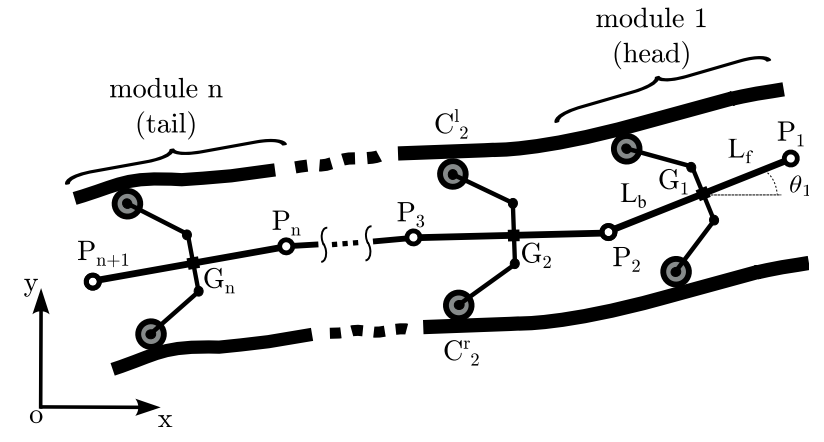

Fig. 1. Kinematic scheme of the articulated mobile robot. 


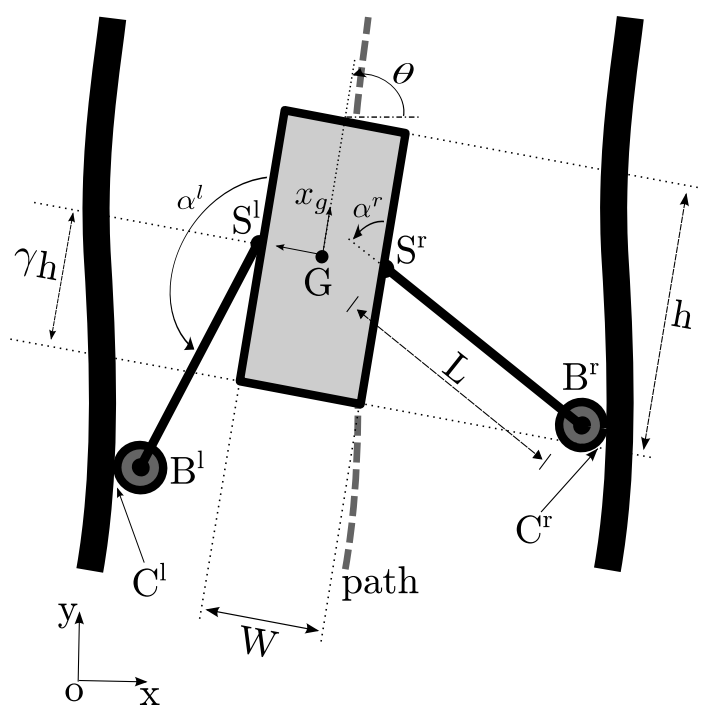

Fig. 2. Schematic of one module with notation. 


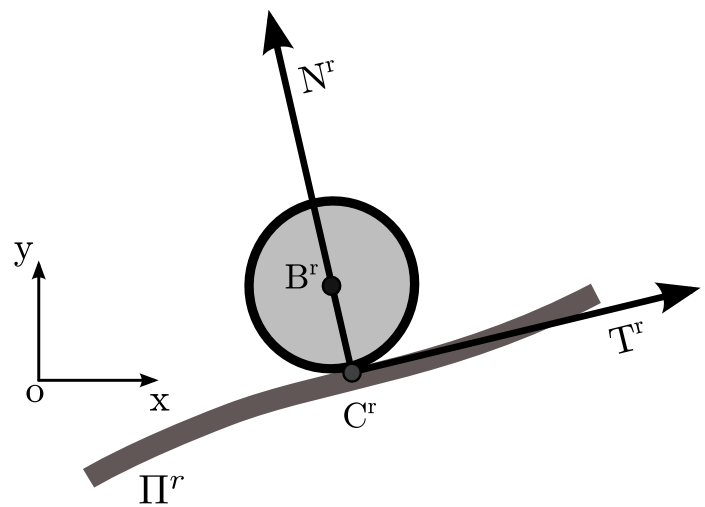

Fig. 3. Right wheel contact with the pipe wall 


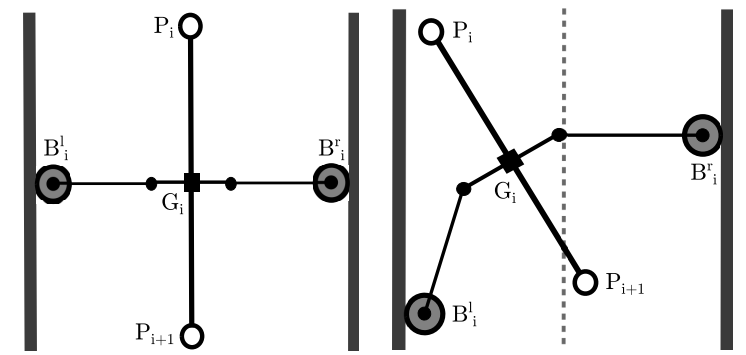

(a)

(b)

Fig. 4. Two serial singularities: (a) both arms are normal to the pipe walls, (b) only one arm is normal to the wall 


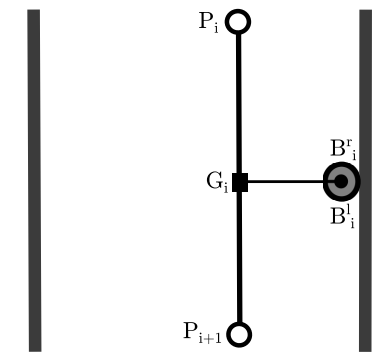

Fig. 5. Parallel singularity 
Path following

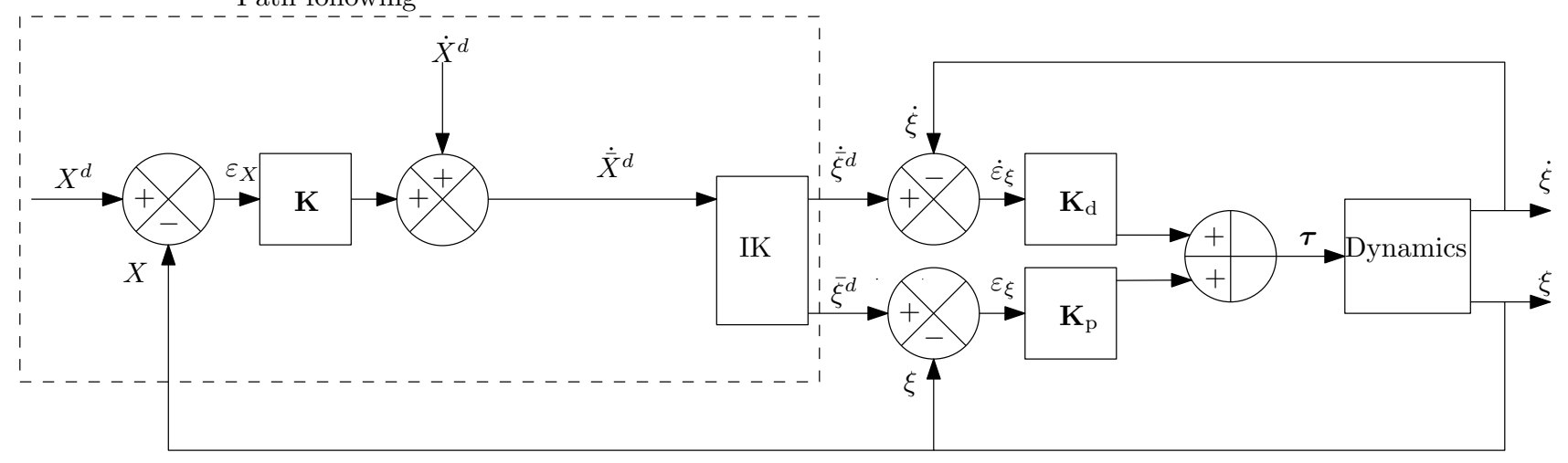

Fig. 6. PD control scheme. "IK" is the acronym of Inverse Kinematics. 


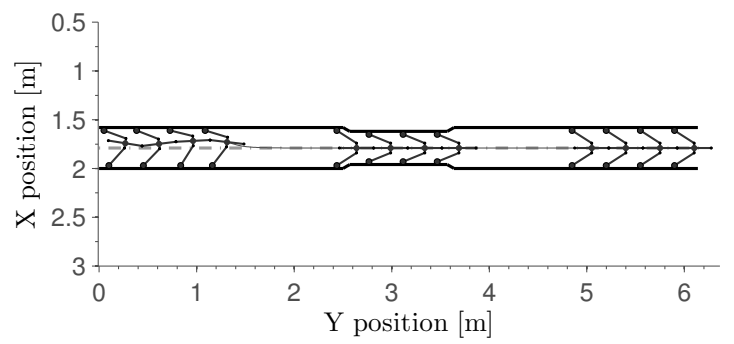

Fig. 7. Key positions in a straight pipe with changing diameter 


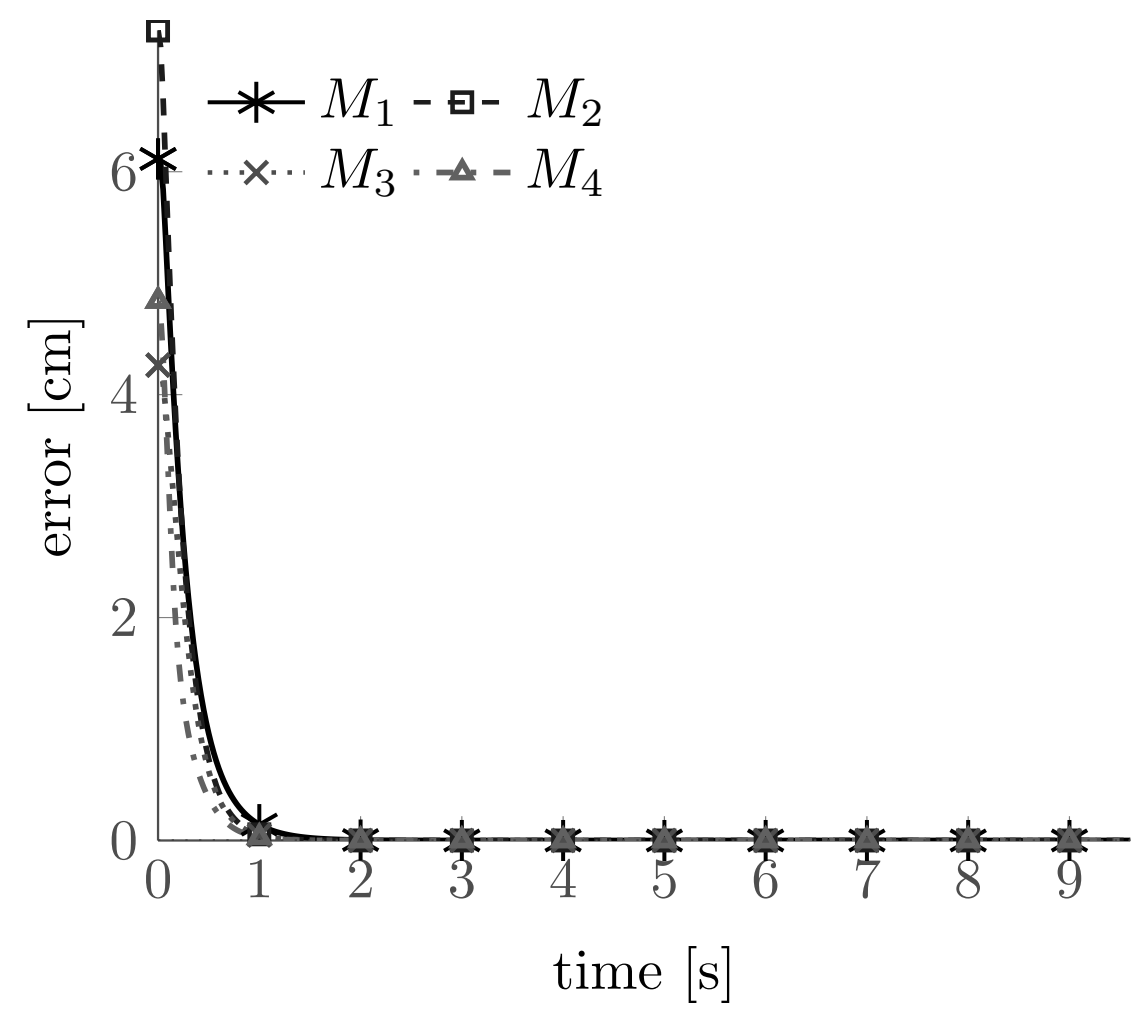

(a) Errors in the position of the centers of mass

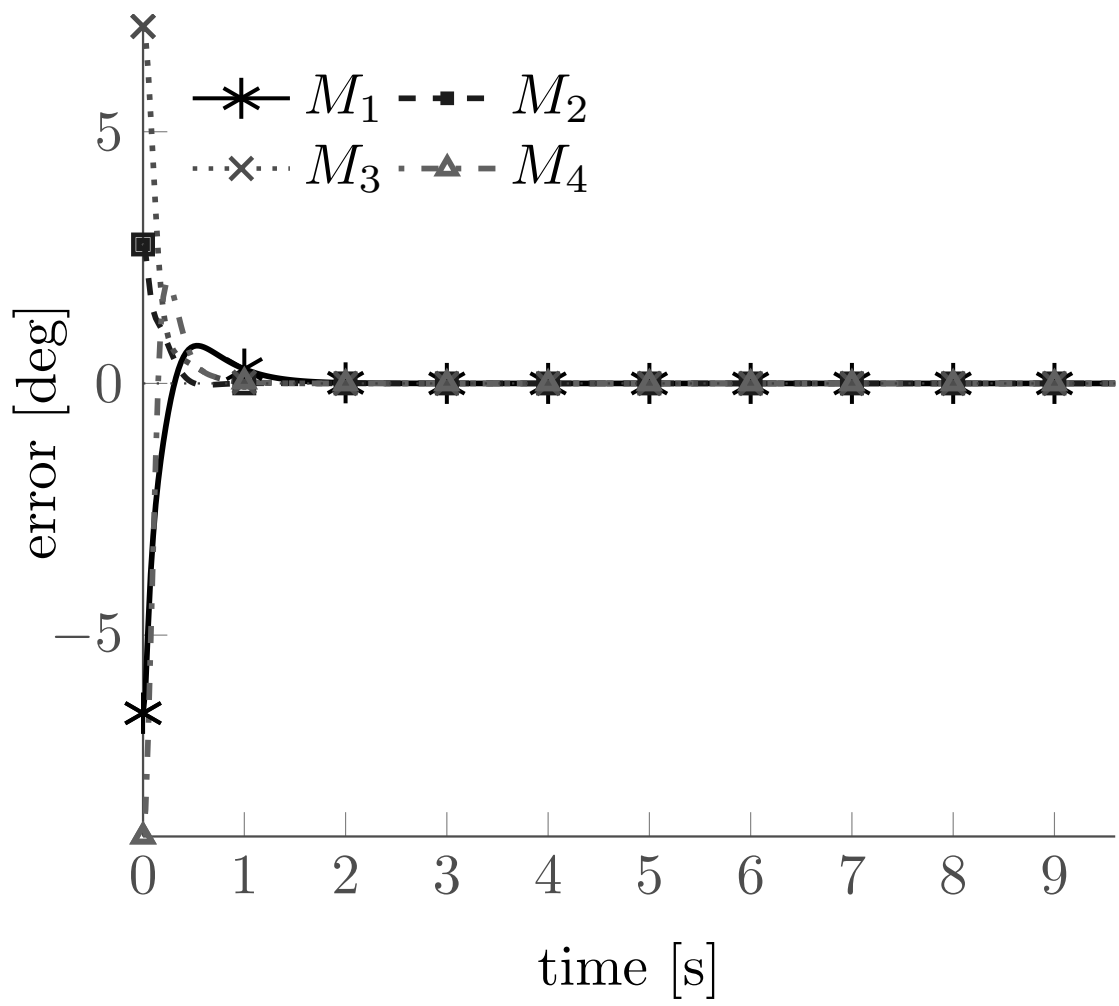

(b) Errors in the orientations

Fig. 8. Linear and angular tracking errors when crossing the straight pipe with changing diameter. $M_{1}, \ldots, M_{4}$ are labels for the modules, assigned progressively from the head $\left(M_{1}\right)$ 


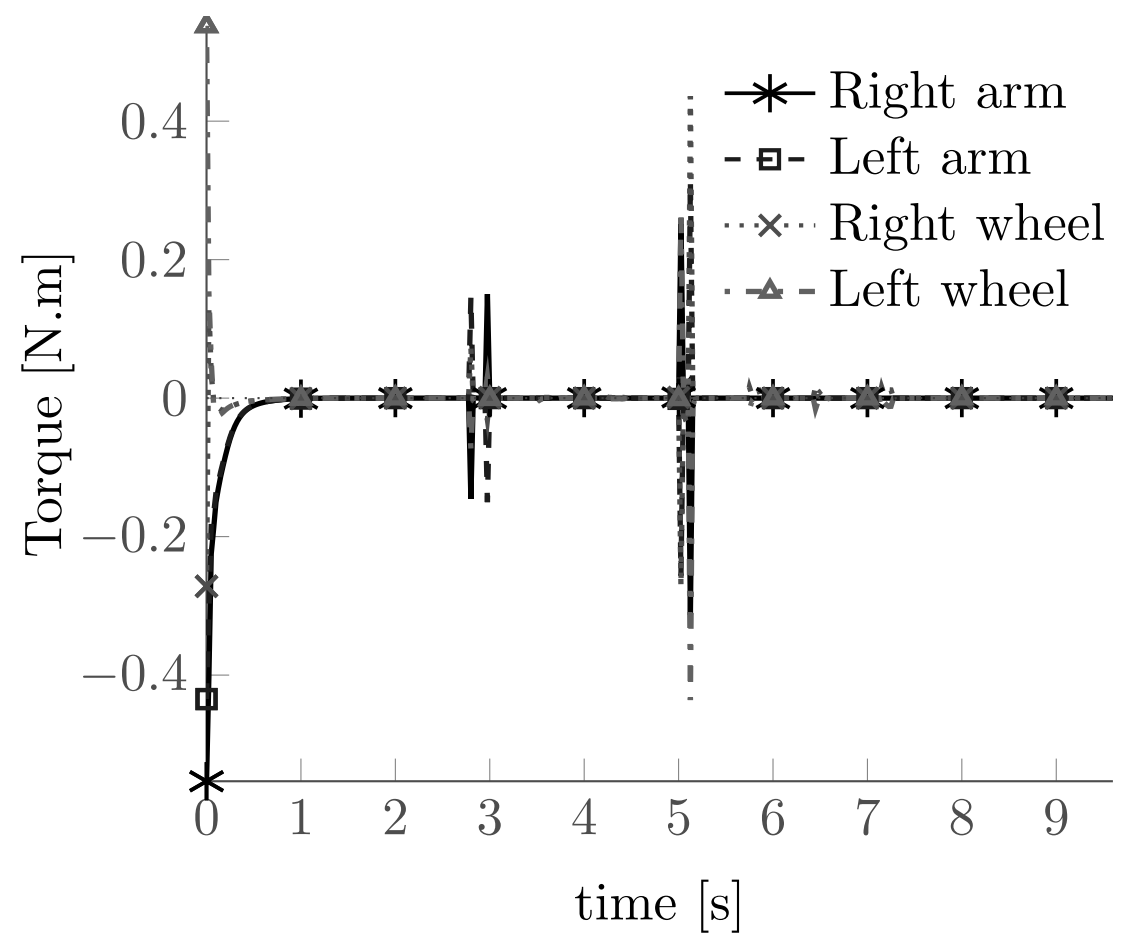

(a) Module 1

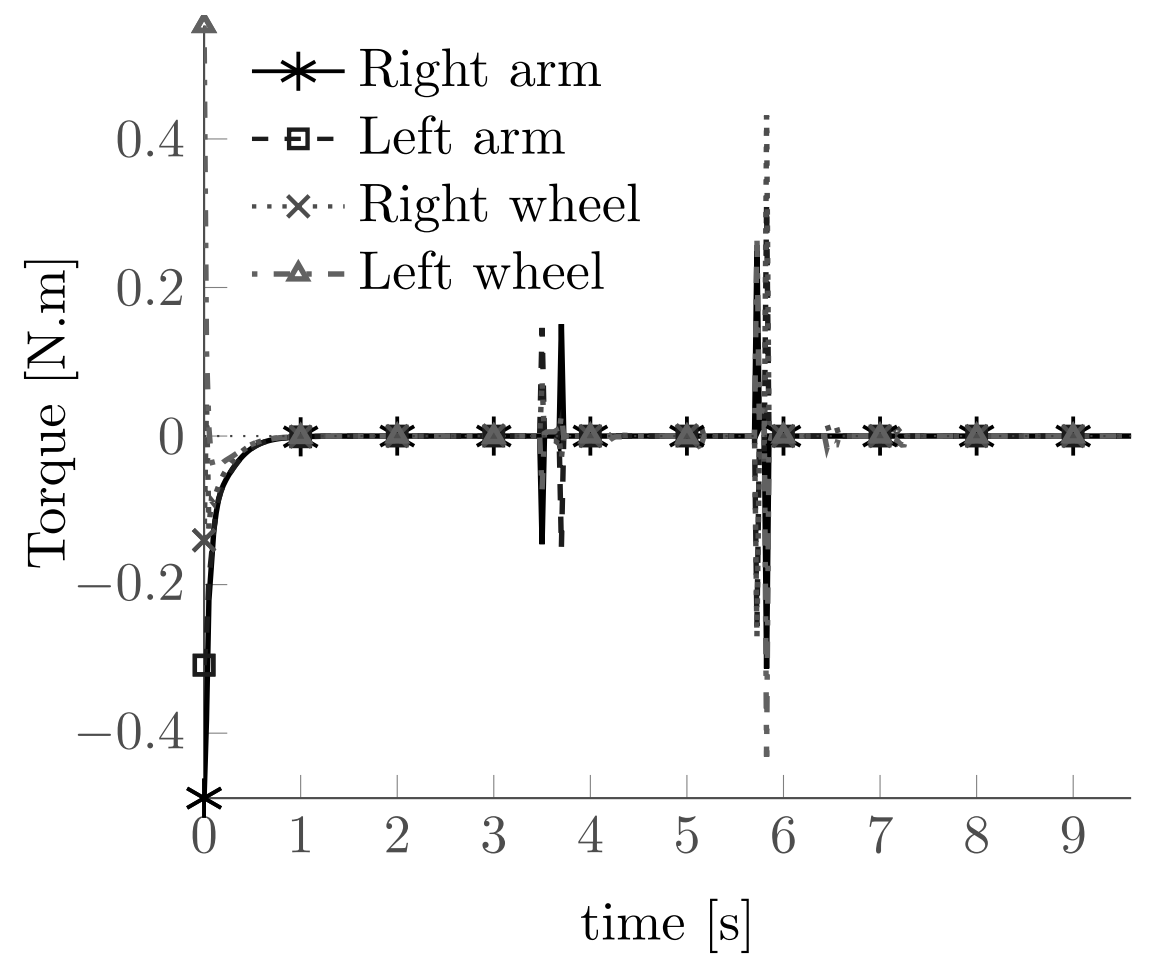

(b) Module 2

Fig. 9. Modules' joint torques: crossing the straight pipe 


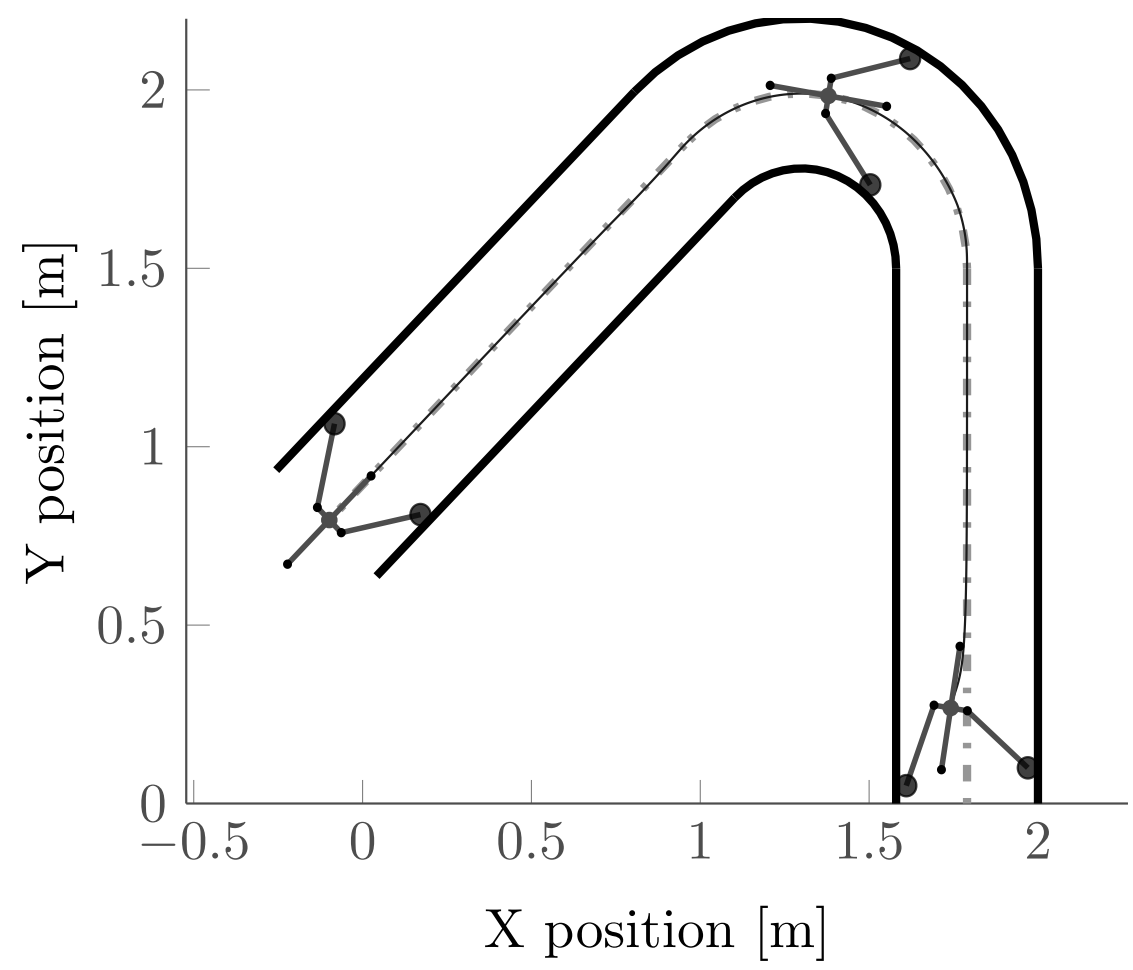

Fig. 10. Initial, intermediate and final positions of a module when crossing a pipe with elbow, while tracking the axis of the pipe 


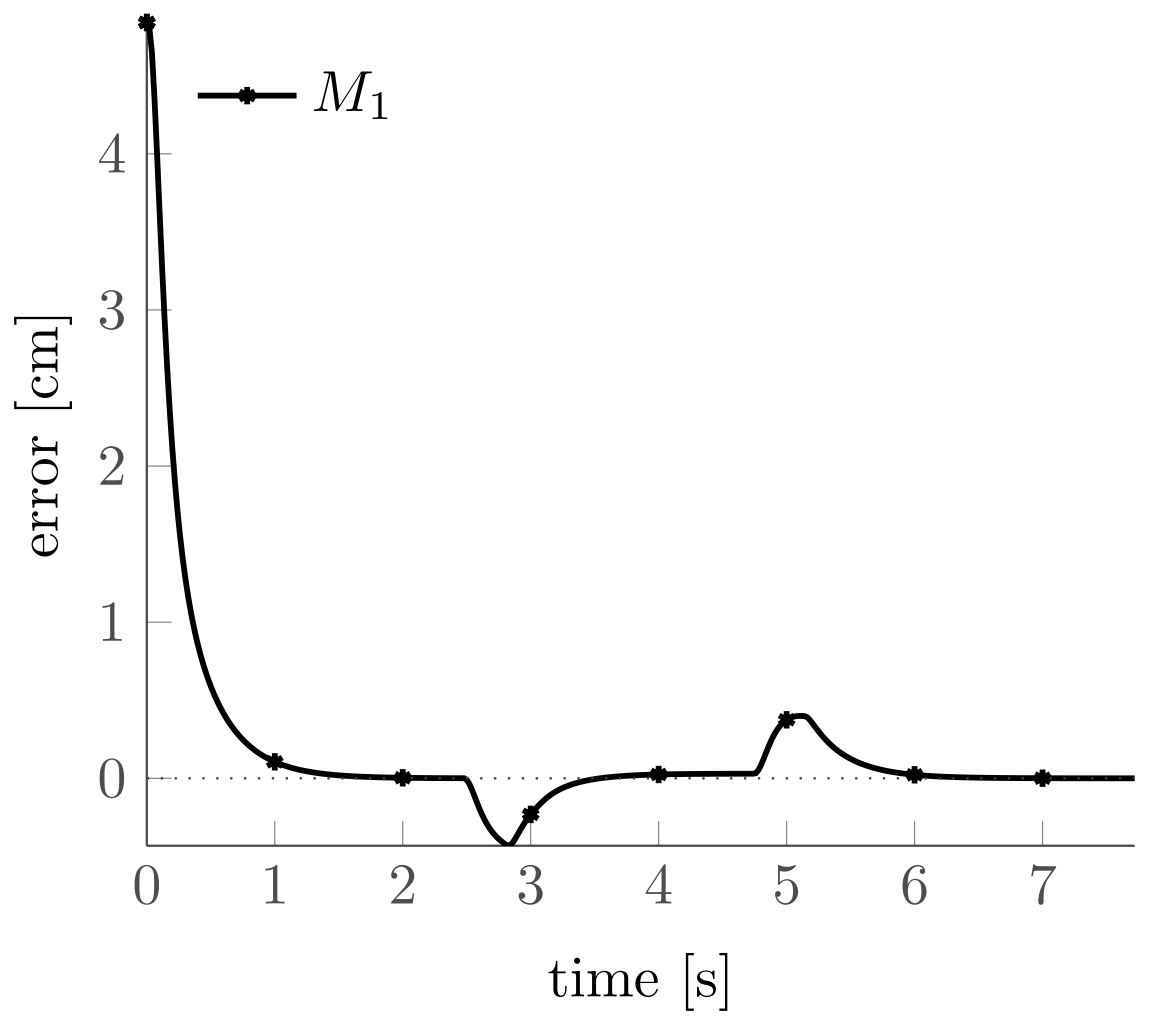

(a) Distance Error

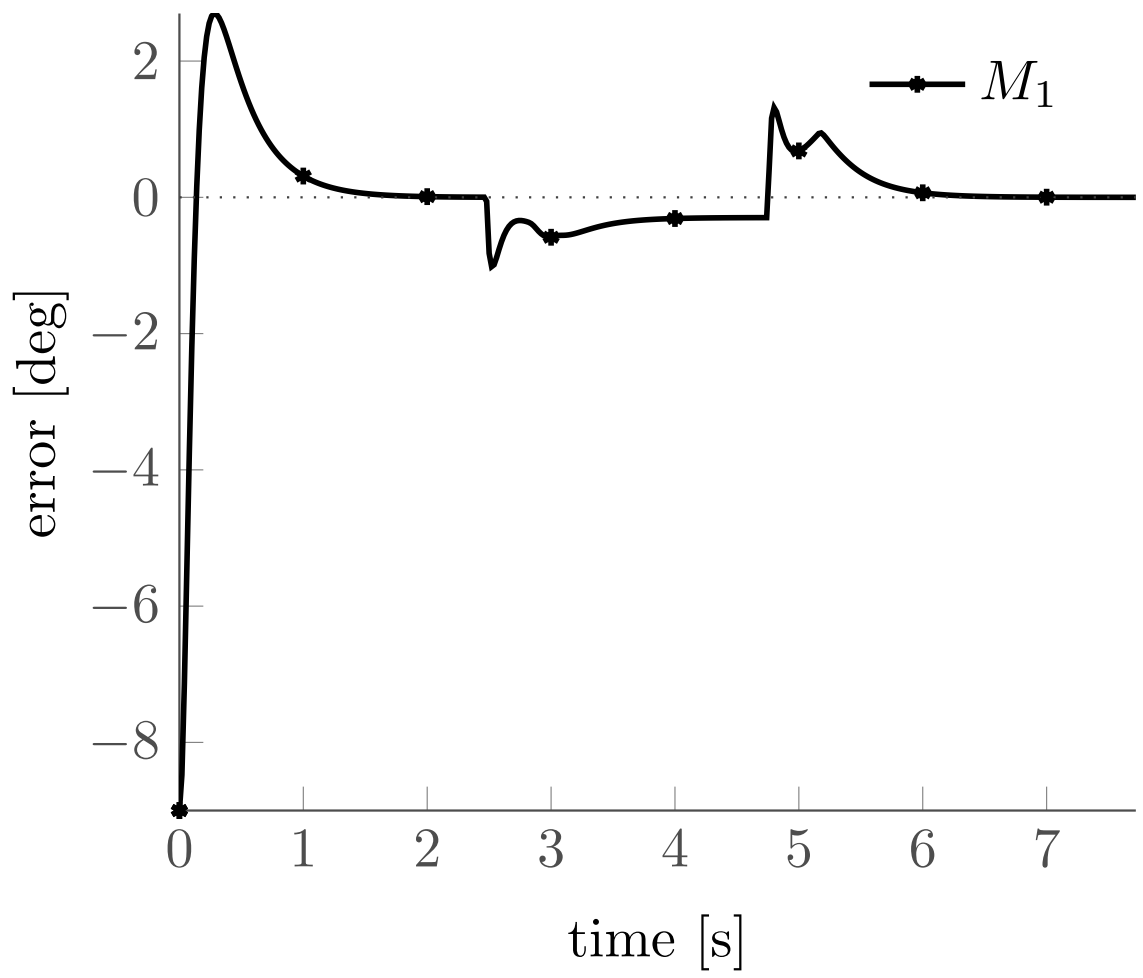

(b) Orientation Error

Fig. 11. Linear and angular tracking errors of a module while tracking the axis of a pipe with elbow. $M_{1}$ labels the only module simulated in this case 


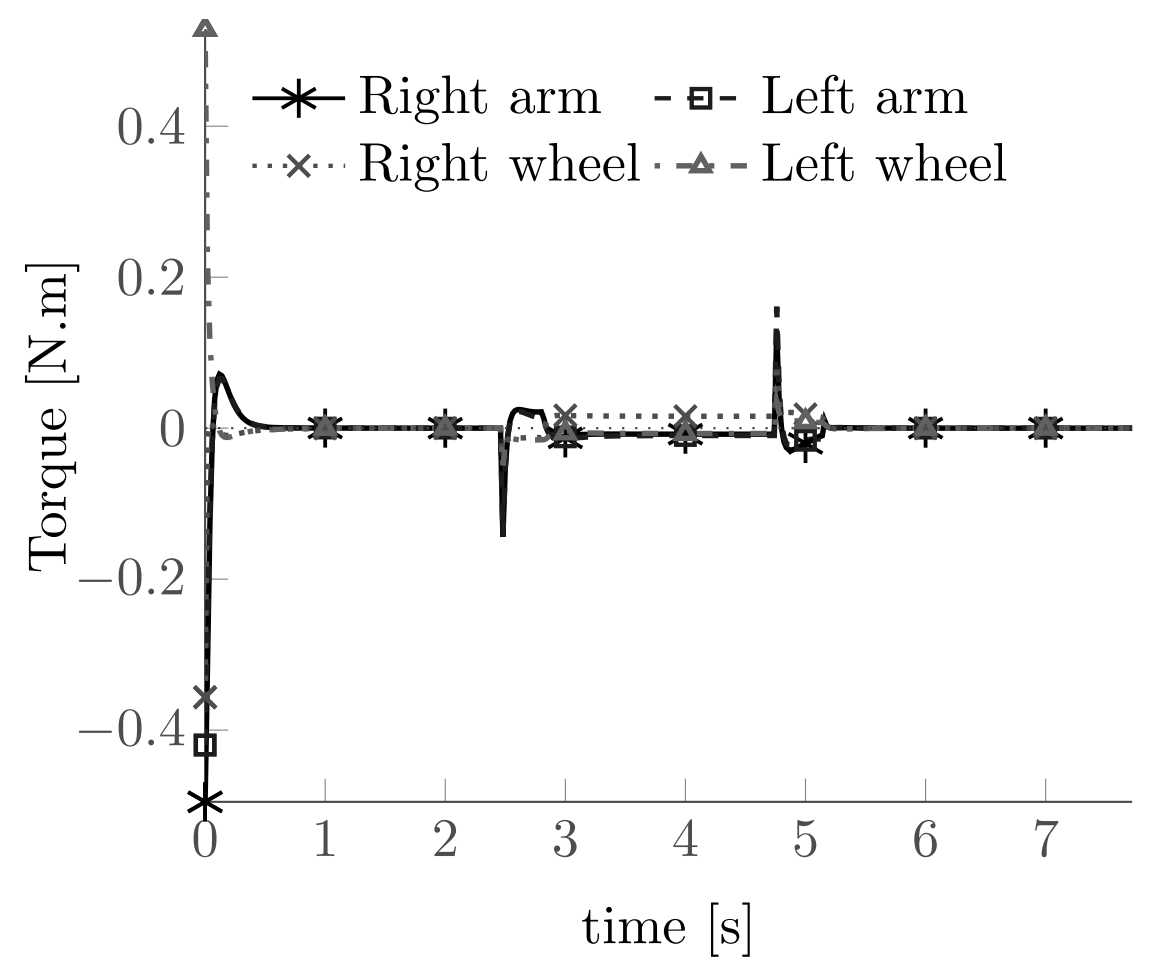

Fig. 12. Joint torques of a module when crossing a pipe with elbow and tracking its axis 


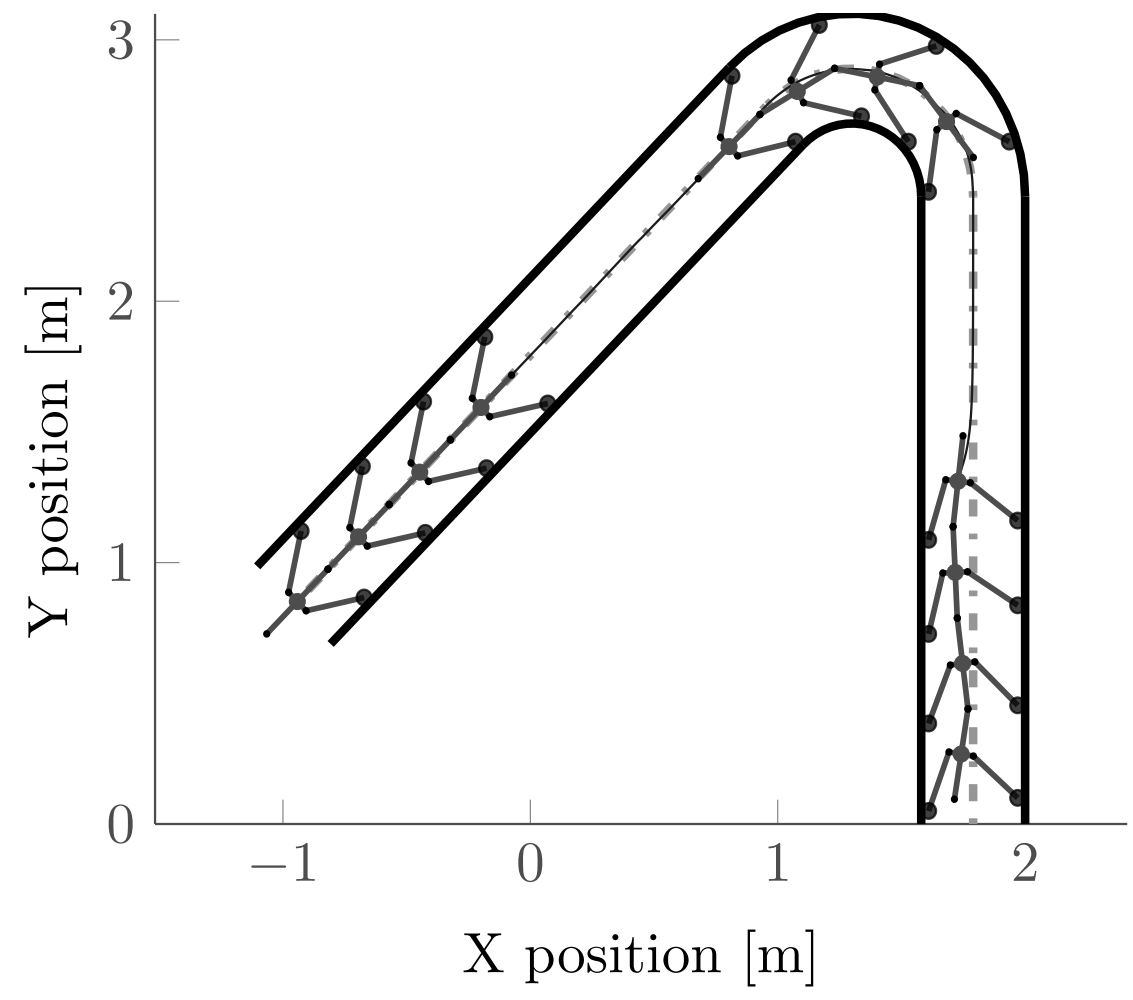

Fig. 13. Three configurations of the articulated vehicle while tracking the axis of a pipe with elbow 


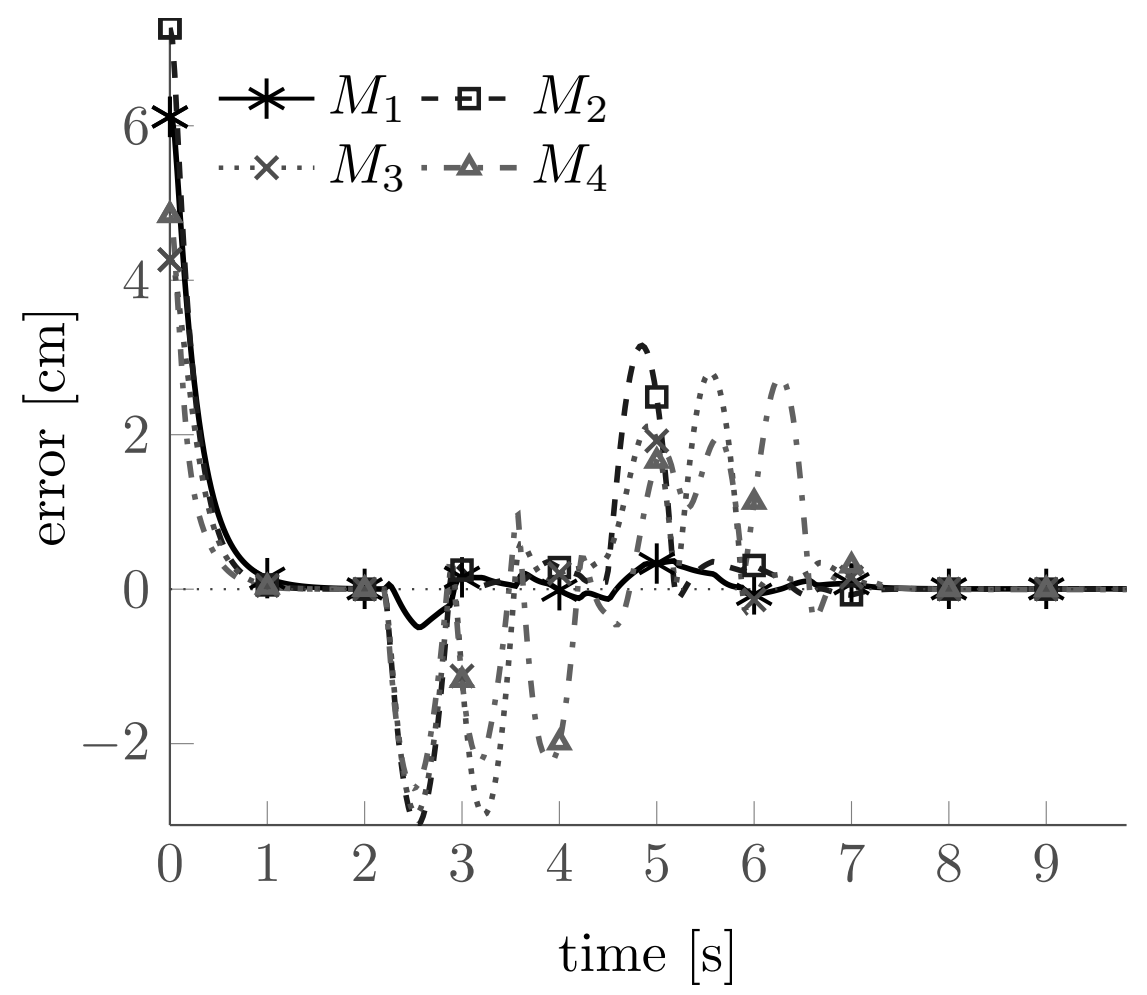

(a) Distance error

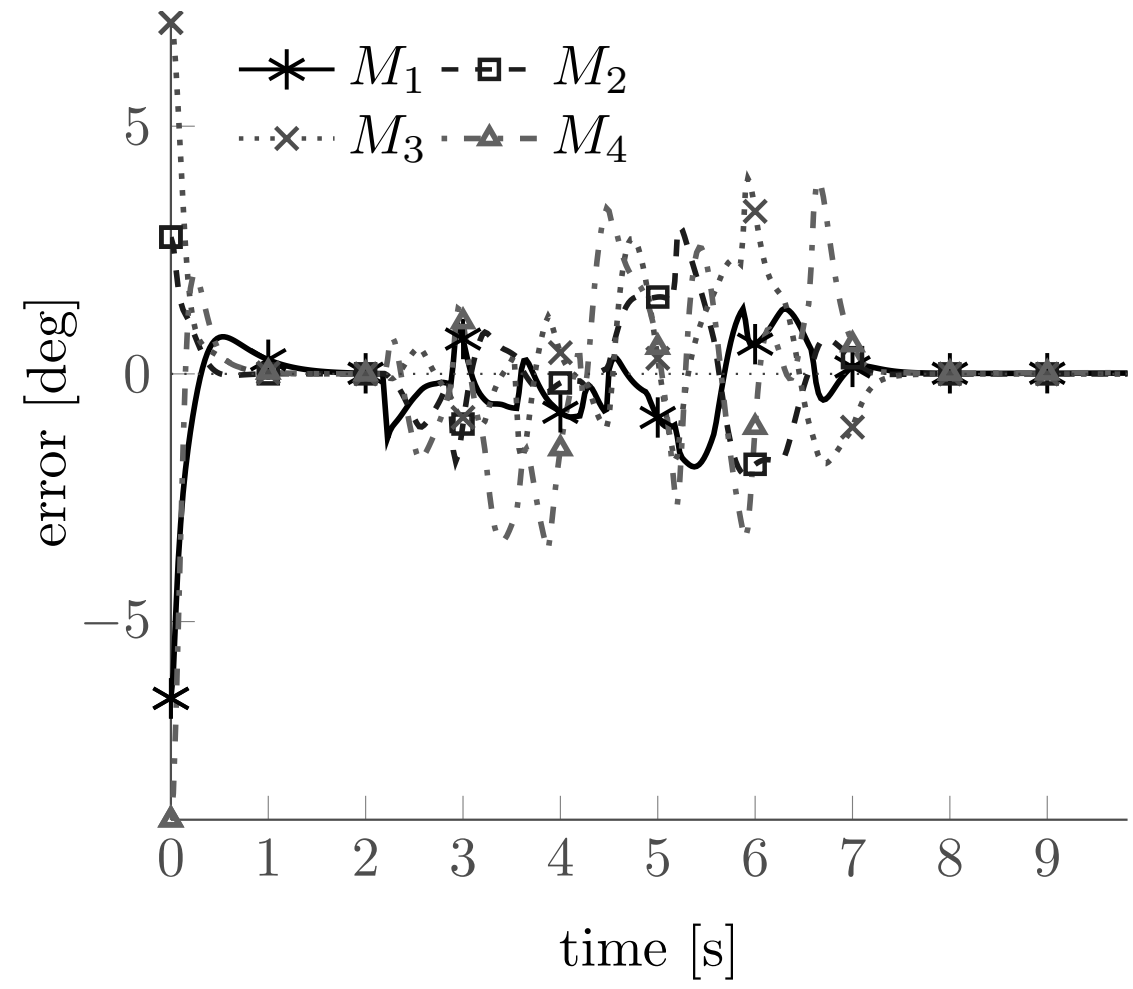

(b) Orientation error

Fig. 14. Linear and angular tracking errors for the articulated vehicle while tracking the axis of a pipe with elbow. $M_{1}, \ldots, M_{4}$ are labels for the modules, assigned progressively from the head $\left(M_{1}\right)$ 


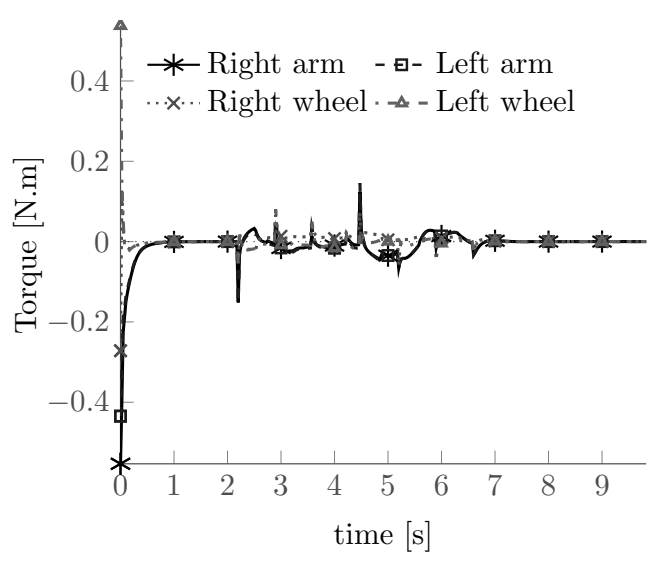

(a) Module 1

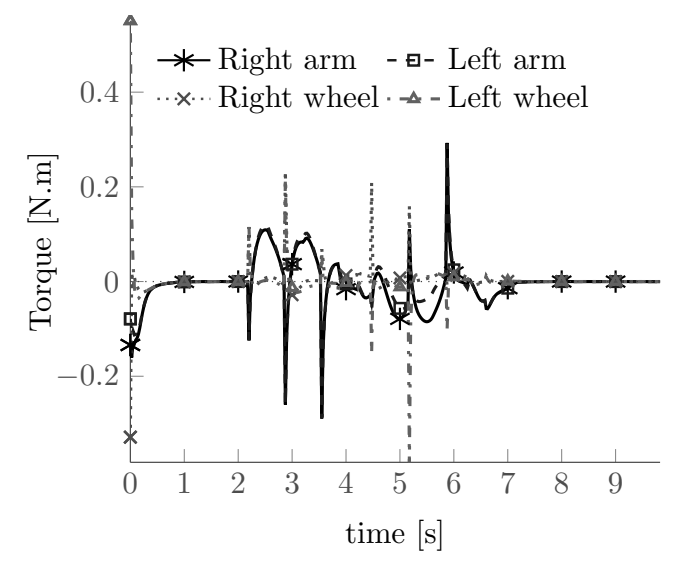

(c) Module 3

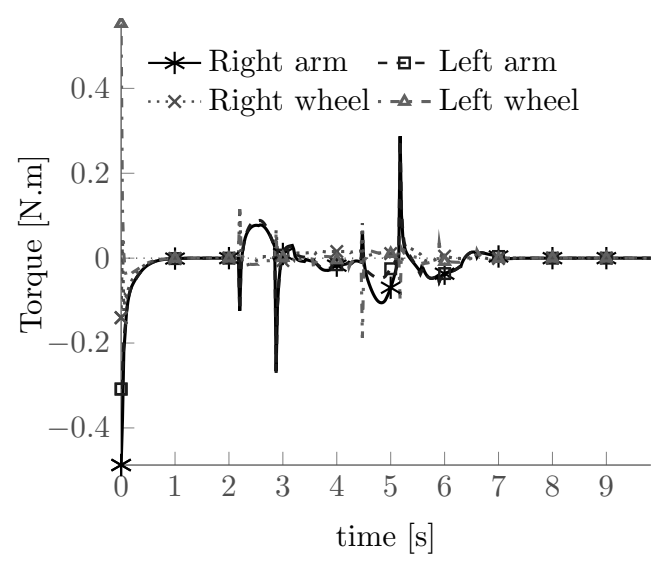

(b) Module 2

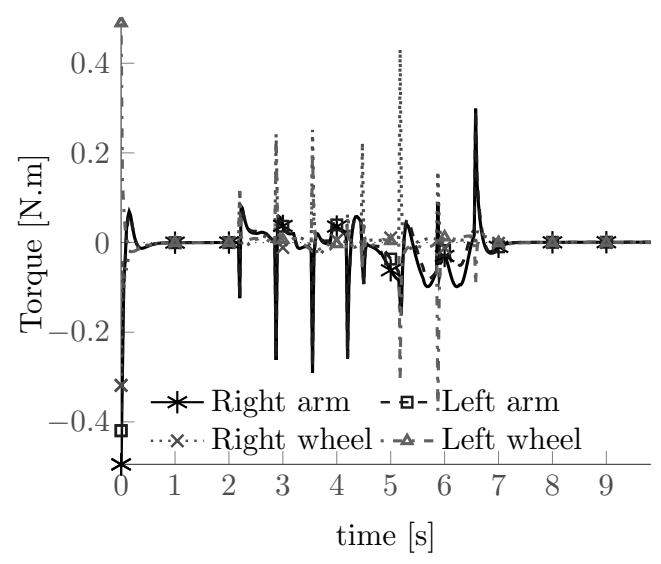

(d) Module 4

Fig. 15. Modules' joint torques: crossing a pipe with elbow 


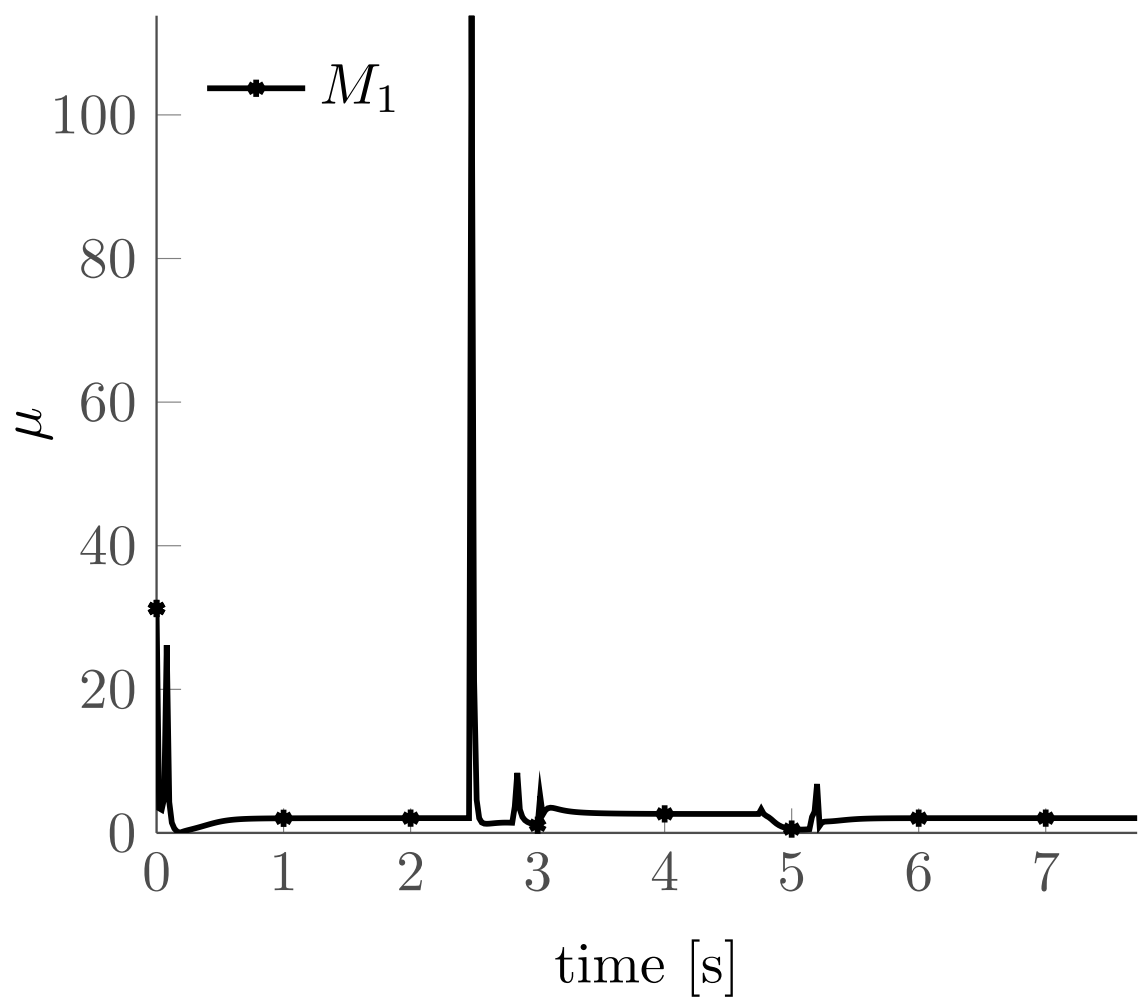

(a) Left wheel

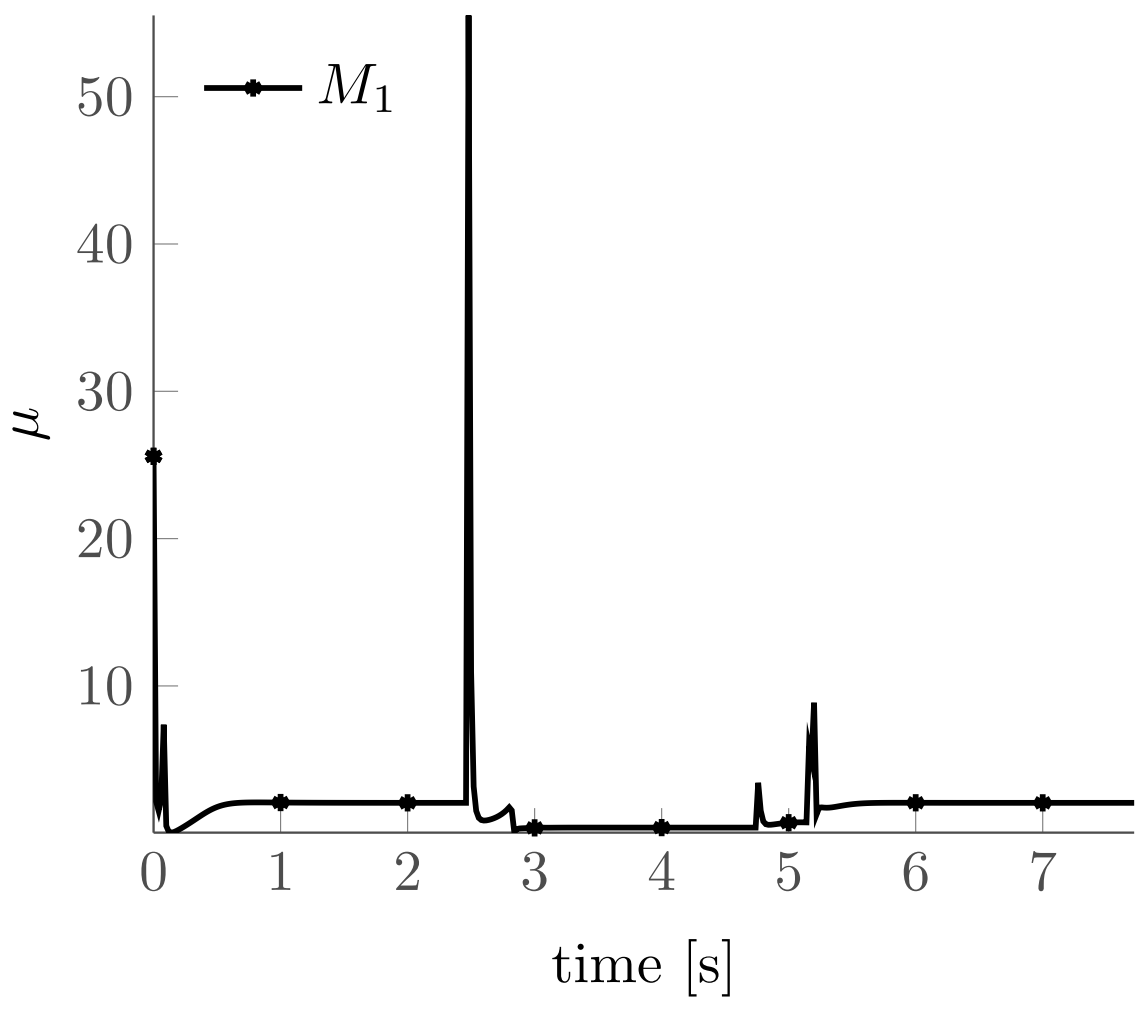

(b) Right wheel

Fig. 16. For one module crossing an elbow: coefficients of friction computed by using the Coulomb friction model, with values computed from Lagrange multipliers. 


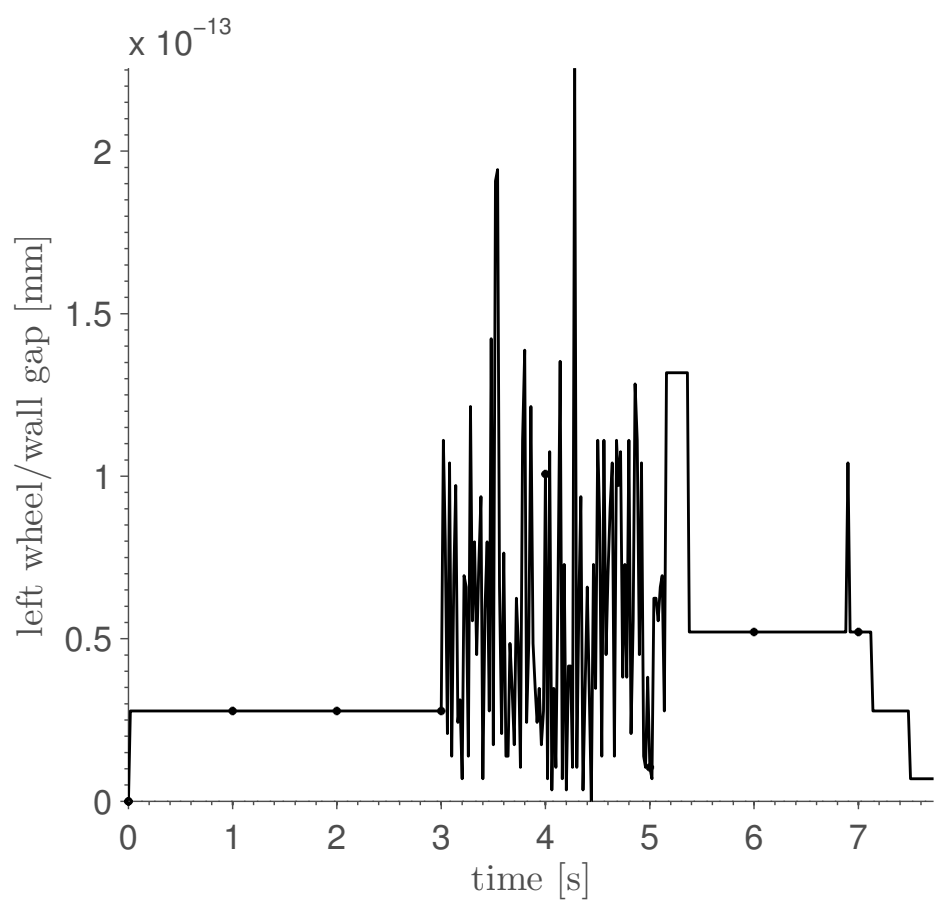

(a) Left wheel

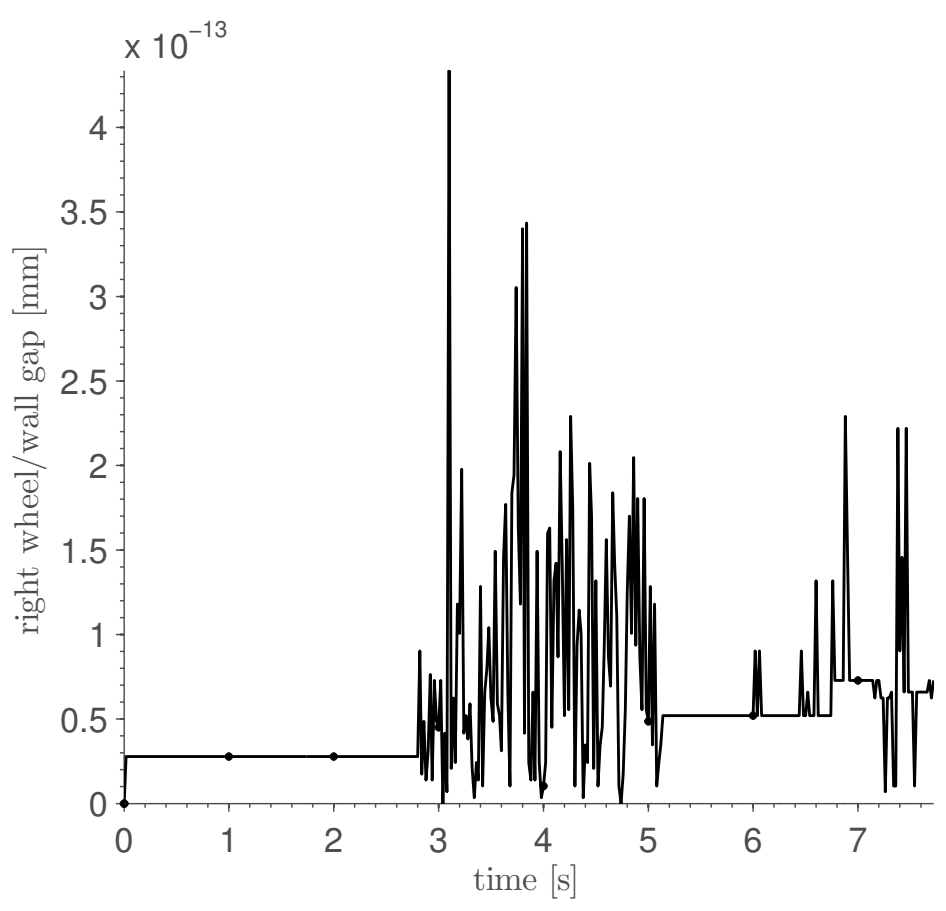

(b) Right wheel

Fig. 17. For one module crossing an elbow: gap between the wall and the wheels. 